\title{
Review \\ A Critical Review on the Economically Feasible and Sustainable Poly(3-Hydroxybutyrate-co-3-hydroxyvalerate) Production from Alkyl Alcohols
}

\author{
Hau Seung Jeremy Wong ${ }^{1,2} \mathbb{D}$, Kesaven Bhubalan ${ }^{3,4}$ and Al-Ashraf Abdullah Amirul $1,2, *$ (D) \\ 1 School of Biological Sciences, Universiti Sains Malaysia, Gelugor 11800, Penang, Malaysia; \\ jeremywong@student.usm.my \\ 2 Centre for Chemical Biology, Universiti Sains Malaysia, Bayan Lepas 11900, Penang, Malaysia \\ 3 Eco-Innovation Research Interest Group, Faculty of Science and Marine Environment, Universiti Malaysia \\ Terengganu, Kuala Nerus 21030, Terengganu, Malaysia; kesaven@umt.edu.my \\ 4 Institute of Marine Biotechnology, Universiti Malaysia Terengganu, Kuala Nerus 21030, Terengganu, Malaysia \\ * Correspondence: amirul@usm.my
}

check for

updates

Citation: Wong, H.S.J.; Bhubalan, K.; Amirul, A.-A.A. A Critical Review on the Economically Feasible and Sustainable Poly(3-Hydroxybutyrateco-3-hydroxyvalerate) Production from Alkyl Alcohols. Polymers 2022, 14, 670. https://doi.org/10.3390/ polym 14040670

Academic Editors: Shengbo Ge, Wanxi Peng and Yequan Sheng

Received: 17 January 2022

Accepted: 5 February 2022

Published: 10 February 2022

Publisher's Note: MDPI stays neutral with regard to jurisdictional claims in published maps and institutional affiliations.

Copyright: () 2022 by the authors Licensee MDPI, Basel, Switzerland. This article is an open access article distributed under the terms and conditions of the Creative Commons Attribution (CC BY) license (https:/ / creativecommons.org/licenses/by/ $4.0 /)$.

\begin{abstract}
Poly(3-hydroxybutyrate-co-3-hydroxyvalerate) ( $\mathrm{P}(3 \mathrm{HB}-c o-3 \mathrm{HV}))$ is the most studied shortchain-length polyhydroxyalkanoates (PHA) with high application importance in various fields. The domination of high-cost propionate and valerate over other 3-hydroxyvalerate $(3 \mathrm{HV})$ precursors owing to their wide preference among PHA-producing bacteria has hindered the development of diverse production processes. As alkyl alcohols are mainly produced from inexpensive starting materials through oxo synthesis, they contribute a cost-effective advantage over propionate and valerate. Moreover, alkyl alcohols can be biosynthesized from natural substrates and organic wastes. Despite their great potential, their toxicity to most PHA-producing bacteria has been the major drawback for their wide implementation as $3 \mathrm{HV}$ precursors for decades. Although the standard PHA-producing bacteria Cupriavidus necator showed promising alcohol tolerance, the $3 \mathrm{HV}$ yield was discouraging. Continuous discovery of alkyl alcohols-utilizing PHA-producing bacteria has enabled broader choices in $3 \mathrm{HV}$ precursor selection for diverse $\mathrm{P}(3 \mathrm{HB}-\mathrm{co}-3 \mathrm{HV})$ production processes with higher economic feasibility. Besides continuous effort in searching for promising wild-type strains, genetic engineering to construct promising recombinant strains based on the understanding of the mechanisms involved in alkyl alcohols toxicity and tolerance is an alternative approach. However, more studies are required for techno-economic assessment to analyze the economic performance of alkyl alcohol-based production compared to that of organic acids.
\end{abstract}

Keywords: 1-pentanol; 1-propanol; 3-hydroxyvalerate precursor; alkyl alcohol tolerance; biosynthesis; oxo synthesis; polyhydroxyalkanoates; poly(3-hydroxybutyrate-co-3-hydroxyvalerate); propionic acid; valeric acid

\section{General Overview}

Polyhydroxyalkanoates (PHA) are emerging as the next generation plastics owing to their plastic-like properties, renewability, biodegradability, and biocompatibility [1]. PHA are accumulated by bacteria under carbon excess but nitrogen-limiting conditions and stored as a reserved energy source in the form of single or multiple granules in the cytoplasm [2]. PHA have gained much industrial interest in the last few decades due to their potential as substitutes for conventional plastics, and various fermentation strategies have been developed to establish microbial PHA production for commercialization. Poly(3-hydroxybutyrate-co-3-hydroxyvalerate) $(\mathrm{P}(3 \mathrm{HB}-\mathrm{co}-3 \mathrm{HV}))$ is the most studied PHA copolymer with mechanical properties comparable to that of polypropylene. The 3hydroxyvalerate $(3 \mathrm{HV})$ monomer provides elastomeric property to the copolymer, enabling broader application compared to the homopolymer poly(3-hydroxybutyrate) $(\mathrm{P}(3 \mathrm{HB}))[3]$. 
The improvement in mechanical properties has paved the way for it to be established for medical, tissue engineering, aquacultural, agricultural, and commodity applications. The commercialization of $\mathrm{P}(3 \mathrm{HB})$ and $\mathrm{P}(3 \mathrm{HB}-\mathrm{co}-3 \mathrm{HV})$ started in the 1970 s by Imperial Chemical Industries, U.K., and Chemie Linz AG, Austria [4]. Currently, P(3HB) and P(3HB-co-3HV) are commercialized by TianAn Biopolymer, China, and Sigma-Aldrich, USA.

Commercialization of PHA is hampered by its high production cost, majorly due to the cost of the carbon feedstock used in microbial fermentation. Over recent decades, various industrial wastes were explored as alternative carbon sources, and numerous mitigation strategies were taken to establish microbial production of $\mathrm{P}(3 \mathrm{HB}-\mathrm{co}-3 \mathrm{HV})$ with high economic feasibility at a commercial scale. Bioconversion of unrelated carbon sources into $\mathrm{P}(3 \mathrm{HB}-\mathrm{co}-3 \mathrm{HV})$ was attempted, but metabolic engineering strategies are generally required to promote precursor-independent pathways to synthesis $\mathrm{P}(3 \mathrm{HB}-c o-3 \mathrm{HV})$, with exceptions for wild types Nocardia or Rhodococcus that can generate propionyl-CoA endogenously from a single carbon source [5-9]. Owing to the relatively simpler practical requirement, $\mathrm{P}(3 \mathrm{HB}-\mathrm{co}-3 \mathrm{HV})$ production from related carbon source(s) remains competitive. Although the employment of wastes contributes to higher economic feasibility, $\mathrm{P}\left(3 \mathrm{HB}-\mathrm{co}_{-}\right.$ $3 \mathrm{HV}$ ) production from a single carbon source has low practicability due to the composition inconsistency of raw components for $3 \mathrm{HV}$ formation [3].

The most common way to incorporate $3 \mathrm{HV}$ monomers is by employing a precursor carbon source as a co-substrate along with the main carbon source that contributes to the 3hydroxybutyrate (3HB) monomer. Precursor carbon sources such as organic acids, alcohols, or some amino acids were studied thoroughly to clarify the metabolic pathways involved and to search for promising precursors of greater potential. Organic acids, especially propionic acid, valeric acid, and their respective salts, are the standard $3 \mathrm{HV}$ precursors owing to their wide acceptance among PHA-producing bacteria. However, organic acids can only be added in low concentrations due to their high toxicity to the bacteria, and their high substrate cost causes lower profitability. Although levulinic acid is way more cost-effective than propionic acid and valeric acid, it seems to be a privilege for Cupriavidus necator, and the production mechanism is yet to be clarified [10,11]. Although some amino acids such as threonine, valine, and isoleucine could be employed as $3 \mathrm{HV}$ precursors, metabolic engineering of the amino acid biosynthetic pathways is required to convert amino acids into propionyl-CoA, which is essential for $3 \mathrm{HV}$ formation. The rare occurrence of alcohols-utilizing ability among PHA-producing bacteria hinders the employment of alkyl alcohols as $3 \mathrm{HV}$ precursors despite their potential as cost-effective substitutes for organic acids [3]. In addition to the merit in lowering the substrate cost, naturally occurring carbon sources such as glucose and glycerol or organic wastes can be converted by microorganisms into alkyl alcohols, thus are promising as cost-effective and sustainable bioresources for $\mathrm{P}(3 \mathrm{HB}-\mathrm{co}-3 \mathrm{HV})$ production [12].

C. necator is the standard PHA-producing bacterium well-known with its wide substrate acceptance range, including alcohols and mercury. Nevertheless, its capability to convert alcohols into PHA is substandard. The $3 \mathrm{HV}$ yield from 1-propanol is low despite its high tolerance toward 1-propanol, and the employment of 1-pentanol results in a remarkably high reduction in C. necator cell biomass and PHA content $[10,13]$. Owing to the economic advantage over organic acids, the employment of alcohols as the $3 \mathrm{HV}$ precursors for $\mathrm{P}(3 \mathrm{HB}-\mathrm{co}-3 \mathrm{HV})$ production was attempted for various bacteria. Interestingly, $\mathrm{P}(3 \mathrm{HB}-$ co-3HV)-producing bacteria favoring alcohols as $3 \mathrm{HV}$ precursors are emerging since the last decade. Since the discovery of Paracoccus denitrificans ATCC 17741 with the capability to convert 1-pentanol into 3HV in 1996, various alkyl alcohol-tolerant PHA-producing bacteria were discovered continually whereby several of them depicted promising $3 \mathrm{HV}$ yield [14].

This critical review condenses the production of $\mathrm{P}(3 \mathrm{HB}-\mathrm{co}-3 \mathrm{HV})$ from alkyl alcohols and the promising potential of alkyl alcohols as cost-effective $3 \mathrm{HV}$ precursors to go beyond the bottleneck in precursors selection that is limited to organic acids. The properties and applications of $\mathrm{P}(3 \mathrm{HB}-\mathrm{co}-3 \mathrm{HV})$ are also discussed. The bioconversion pathways of 
1-propanol and 1-pentanol into $3 \mathrm{HV}$ with respect to propionic acid and valeric acid are visualized, and the performance of discovered alkyl alcohol-tolerant PHA-producing bacteria is highlighted. Oxo synthesis and biosynthesis of 1-propanol and 1-pentanol from natural substrates as well as organic wastes were described. Furthermore, the mode of action of alkyl alcohols on bacterial proteins and the bacterial mechanisms involved in response to alcoholic stress are also discussed. The strategies for wide implementation of alkyl alcohols for $\mathrm{P}(3 \mathrm{HB}-\mathrm{co}-3 \mathrm{HV})$ production and the challenges ahead are highlighted as well to comment on the potential of alkyl alcohols as the next generation $3 \mathrm{HV}$ precursors.

\section{2. $\mathrm{P}(3 \mathrm{HB}-\mathrm{co}-3 \mathrm{HV})$ Properties and Applications}

$\mathrm{P}(3 \mathrm{HB})$ is a relatively stiff and brittle polyester with poor elongation at break [15]. It is a fragile material, and its mechanical properties deteriorate with time due to secondary crystallization accompanied by aging at room temperature, which is the major cause of its brittleness [16]. Although the lack of elasticity causes a drawback in its application as packaging materials, its high mechanical properties are applicable as bone tissues aid in supporting body weight. $\mathrm{P}(3 \mathrm{HB})$ facilitates reconstructive osteogenesis. $\mathrm{P}(3 \mathrm{HB})$ and its biocomposite incorporated with $20 \mathrm{wt} \%$ hydroxyapatite, which makes up $65-70 \%$ of the bone matrix, show pronounced osteoplastic properties owing to their slow degradation that corresponds to the growth of new bones. Powdered $\mathrm{P}(3 \mathrm{HB})$ and $\mathrm{P}(3 \mathrm{HB}) /$ tienam are excellent antibacterial bone filling materials that contribute to 1-fold lower growth and complete growth inhibition of Staphylococcus aureus post surgery, respectively [17].

The incorporation of the $\mathrm{C}_{5} 3 \mathrm{HV}$ monomer into $\mathrm{P}(3 \mathrm{HB})$ results in $\mathrm{P}(3 \mathrm{HB}-\mathrm{co}-3 \mathrm{HV})$ with decreased crystallinity, thus leading to decreased stiffness, decreased brittleness, and enhanced biodegradability compared to that of $\mathrm{P}(3 \mathrm{HB})$ [18]. The properties of $\mathrm{P}(3 \mathrm{HB}-\mathrm{co}-3 \mathrm{HV})$ are dependent on the ratio of the two monomers where the $3 \mathrm{HB}$ monomer contributes stiffness, and the $3 \mathrm{HV}$ monomer contributes flexibility to the copolymer. The composition of the $3 \mathrm{HV}$ monomer determines the defection of the $\mathrm{P}(3 \mathrm{HB})$ lamellae crystals, leading to the disruption of its crystallinity and resulting in improved polymer flexibility (Figure 1) [19]. The lower degree of crystallinity and melting point of $\mathrm{P}(3 \mathrm{HB}-\mathrm{co}-3 \mathrm{HV})$ lead to a higher degradation rate that is directly proportional to the molar fraction of $3 \mathrm{HV}$ of the copolymer compared to that of $\mathrm{P}(3 \mathrm{HB})$ [18]. The $3 \mathrm{HV}$ fraction contributes to a greater amorphous region for enzymatic attacks that leads to enhanced and adjustable biodegradability for applications such as implants for bone support, stents for artery support in angioplasty, and drug delivery carriers. Although $\mathrm{P}(3 \mathrm{HB}-\mathrm{co}-3 \mathrm{HV})$ has a 2-fold lower maximum water permeability than poly(lactic acid) which is another biodegradable aliphatic polyester of great biotechnological importance, causing lower hydrolytic degradation due to lower water uptake, the degradation rate of $\mathrm{P}(3 \mathrm{HB}-\mathrm{co}-3 \mathrm{HV})$-based biomedical devices are adjustable with molar fraction of 3HV [18,20,21]. Hydrophilic poly(ethylene glycol) and monomethoxy poly(ethylene glycol) can also be incorporated into $\mathrm{P}(3 \mathrm{HB}-\mathrm{co}-3 \mathrm{HV})$ to form nanoparticles with a hydrophilic outer layer and a hydrophobic inner layer for improved chemical functionalization and compatibility with therapeutic drugs besides benefiting drug release control [22-24]. Incorporation of other desired properties for biomedical applications can also be achieved (Table 1). 


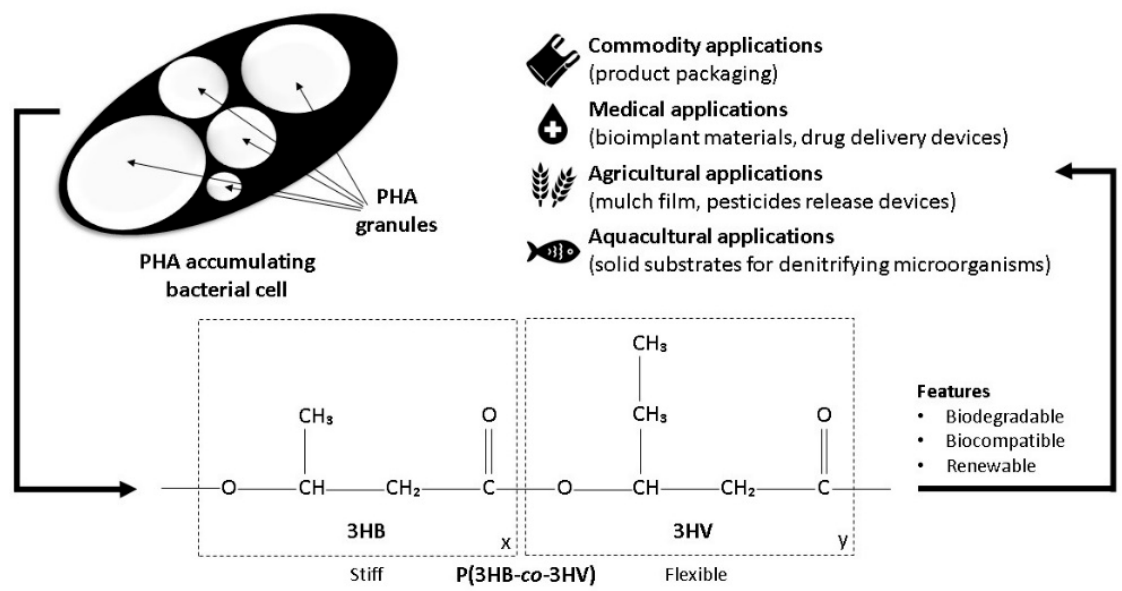

Figure 1. Microbial PHA granule, $\mathrm{P}(3 \mathrm{HB}-\mathrm{co}-3 \mathrm{HV})$ structure, and applications.

Table 1. Properties improvement after the incorporation of a secondary (and tertiary) component into $\mathrm{P}(3 \mathrm{HB}-\mathrm{co}-3 \mathrm{HV})$ and their potential applications.

\begin{tabular}{|c|c|c|c|}
\hline Incorporated Components $\mathrm{A}$ & Changes in the Properties & $\begin{array}{c}\text { Potential } \\
\text { Applications }\end{array}$ & Ref. \\
\hline $\begin{array}{l}\alpha-\mathbf{P}(\mathbf{3 H B}) \\
\text { Incorporation method: } \\
\text { Solvent casting } \\
\text { 3HV fraction: } 10 \mathrm{~mol} \%\end{array}$ & $\begin{array}{c}\mathrm{P}(3 \mathrm{HB}-\mathrm{co}-3 \mathrm{HV}): \alpha-\mathrm{P}(3 \mathrm{HB})(100: 0 \rightarrow 50: 50) \\
\text { Melting temperature: } 145 \rightarrow 133{ }^{\circ} \mathrm{C} \\
\text { Degree of crystallinity: } 61 \% \rightarrow 30 \% \\
\text { Tensile strength: } 27 \rightarrow 7 \mathrm{MPa} \\
\text { Elongation at break: } 1 \% \rightarrow 29 \% \\
\text { Young's modulus: } 1500 \rightarrow 240 \mathrm{MPa} \\
\text { Enzymatic degradation: } 85 \% \rightarrow 94 \%\end{array}$ & $\begin{array}{c}\text { Packaging } \\
\text { material }\end{array}$ & [25] \\
\hline $\begin{array}{l}\text { AS } \\
\text { Incorporation method: } \\
\text { Solvent casting } \\
\text { 3HV fraction: } 59 \mathrm{~mol} \%\end{array}$ & $\begin{array}{c}\mathrm{P}(3 \mathrm{HB}-\mathrm{co}-3 \mathrm{HV}) / \mathrm{P}(3 \mathrm{HB}-\mathrm{co}-3 \mathrm{HV}): \mathrm{AS} \\
\text { Melting temperature: } 275.84^{\circ} \mathrm{C} / 294.97{ }^{\circ} \mathrm{C} \\
\text { Degree of crystallinity: } 98.96 \% / 98.23 \% \\
\text { Free radical scavenging activity }(24 \mathrm{~h}): 1 \% / 14 \% \\
\text { Incubation biodegradation (day 6): } \\
\text { smooth surface/small pits }\end{array}$ & $\begin{array}{c}\text { Therapeutic } \\
\text { implant }\end{array}$ & [26] \\
\hline $\begin{array}{l}\text { CNC } \\
\text { Incorporation method: } \\
\text { Solvent casting } \\
\text { 3HV fraction: } 12 \mathrm{~mol} \%\end{array}$ & $\begin{array}{c}\text { P(3HB-co-3HV):CNC }(100: 0 \rightarrow 94: 6) \\
\text { Melting temperature: } 136.8 \rightarrow 151.1^{\circ} \mathrm{C} \\
\text { Crystallization temperature: } 96.5 \rightarrow 101.2^{\circ} \mathrm{C} \\
\text { Degree of crystallinity: } 49.9 \% \rightarrow 57.5 \% \\
\text { Water vapor transmission rate: } 308 \rightarrow 115 \mathrm{~g} \mathrm{~m}^{-2} \text { day }^{-1} \\
\text { Oxygen transfer rate: } 425 \rightarrow 113 \mathrm{~cm} \mathrm{~m}^{-2} \text { day }^{-1}\end{array}$ & $\begin{array}{c}\text { Packaging } \\
\text { material }\end{array}$ & [27] \\
\hline $\begin{array}{l}\text { DDGS or Misc } \\
\text { Incorporation method: } \\
\text { Twin screw extrusion } \\
\text { 3HV fraction: } 5 \text { mol\% }\end{array}$ & 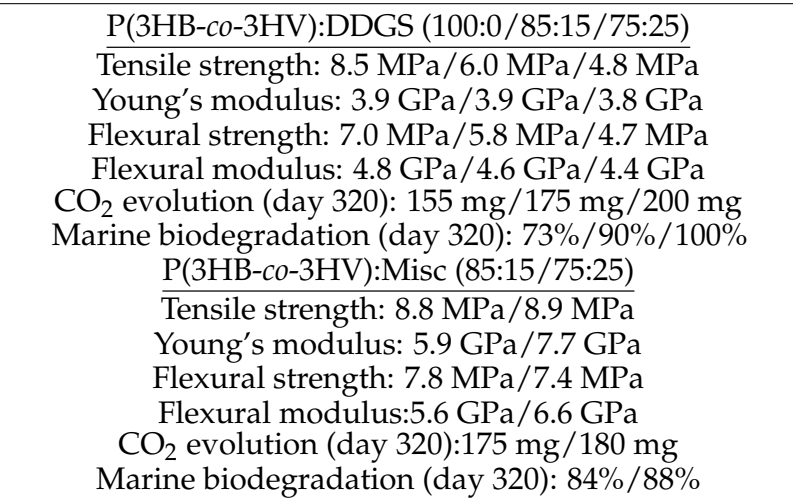 & $\begin{array}{l}\text { Packaging } \\
\text { material }\end{array}$ & [28] \\
\hline
\end{tabular}


Table 1. Cont.

\begin{tabular}{|c|c|c|c|}
\hline Incorporated Components ${ }^{A}$ & Changes in the Properties & $\begin{array}{c}\text { Potential } \\
\text { Applications }\end{array}$ & Ref. \\
\hline $\begin{array}{l}\text { Eugenol } \\
\text { Incorporation method: } \\
\text { Electrospinning } \\
\text { 3HV fraction: } 3 \mathrm{~mol} \%\end{array}$ & $\begin{array}{c}\text { P(3HB-co-3HV):Eugenol }(100: 0 \rightarrow 85: 15) \\
\text { Temperature of } 5 \% \text { weight loss: } 276.6 \rightarrow 160.8^{\circ} \mathrm{C} \\
\text { Degradation temperature: } 304.7 \rightarrow 293.3^{\circ} \mathrm{C} \\
\text { Mass loss at degradation temperature: } 61.01 \% \rightarrow 76.36 \% \\
\text { Water vapor permeability: } \\
4.05 \times 10^{14} \rightarrow 0.95 \times 10^{14} \mathrm{Kg} \mathrm{m} \mathrm{m}^{-2} \mathrm{~s}^{-1} \mathrm{~Pa}^{-1} \\
\text { Limonene vapor permeability: } 3.75 \rightarrow 0.81 \mathrm{Kg} \mathrm{m} \mathrm{m}^{-2} \mathrm{~s}^{-1} \mathrm{~Pa}^{-1} \\
\text { Water vapor permeance: } 5.87 \rightarrow 1.33 \mathrm{Kg} \mathrm{m} \mathrm{m}^{-2} \mathrm{~s}^{-1} \mathrm{~Pa}^{-1} \\
\text { Limonene vapor permeance: } 5.44 \rightarrow 1.14 \mathrm{Kg} \mathrm{m} \mathrm{m}^{-2} \mathrm{~s}^{-1} \mathrm{~Pa}^{-1} \\
\text { Tensile strength: } 1252 \rightarrow 1897 \mathrm{MPa} \\
\text { Elongation at break: } 2.0 \% \rightarrow 2.5 \% \\
\text { Young's modulus: } 18.1 \rightarrow 26.5 \mathrm{MPa} \\
\text { S. aureus growth: } 5.16 \rightarrow 3.45 \log \left(\mathrm{CFU} \mathrm{mL}{ }^{-1}\right) \\
\text { Escherichia coli growth: } 5.79 \rightarrow 3.88 \log \left(\mathrm{CFU} \mathrm{mL} \mathrm{m}^{-1}\right)\end{array}$ & $\begin{array}{l}\text { Antimicrobial } \\
\text { food } \\
\text { packaging }\end{array}$ & [29] \\
\hline $\begin{array}{l}\text { HA } \\
\text { Incorporation method: } \\
\text { Melt-pressing } \\
\text { 3HV fraction: } 8-24 \text { mol\% }\end{array}$ & $\begin{array}{c}\frac{\mathrm{P}(3 \mathrm{HB}-\mathrm{co}-3 \mathrm{HV}), 0 \rightarrow 24 \mathrm{~mol} \% 3 \mathrm{HV}}{\text { Melting temperature: } 170 \rightarrow 129{ }^{\circ} \mathrm{C}} \\
\text { Degree of crystallinity: } 69 \% \rightarrow 55 \% \\
\mathrm{P}(3 \mathrm{HB}-\mathrm{co}-3 \mathrm{HV}): \mathrm{HA}(30: 70), 0 \rightarrow 24 \mathrm{~mol} \% 3 \mathrm{HV} \\
\text { Tensile strength: } 67 \rightarrow 23 \mathrm{MPa} \\
\text { Elongation at break: } 2.65 \% \rightarrow 3.84 \% \\
\text { Young's modulus: } 2.52 \rightarrow 0.47 \mathrm{GPa}\end{array}$ & $\begin{array}{l}\text { Bone } \\
\text { implant }\end{array}$ & {$[30]$} \\
\hline $\begin{array}{l}\text { MAT } \\
\text { Incorporation method: } \\
\text { Solvent casting } \\
\text { 3HV fraction: } 4 \text { mol } \%\end{array}$ & $\begin{array}{c}\text { P(3HB-co-3HV):MAT }(100: 0 \rightarrow 95: 5) \\
\text { Melting temperature: } 168.58 \rightarrow 130.91{ }^{\circ} \mathrm{C} \\
\text { Glass transition temperature: }-2.03 \rightarrow-6.61^{\circ} \mathrm{C} \\
\text { Crystallization temperature: } 46.15 \rightarrow 46.98^{\circ} \mathrm{C} \\
\text { Degree of crystallinity: } 53.7 \% \rightarrow 36.8^{\circ} \%\end{array}$ & $\begin{array}{l}\text { Packaging } \\
\text { material }\end{array}$ & [31] \\
\hline $\begin{array}{l}\text { MCPA } \\
\text { Incorporation method: } \\
\text { Melt-blending and } \\
\text { hot-pressing } \\
\text { 3HV fraction: } 3 \text { mol\% }\end{array}$ & $\begin{array}{c}\mathrm{P}(3 \mathrm{HB}-\mathrm{co}-3 \mathrm{HV})-\mathrm{MCPA}(95: 5 / 90: 10 / 85: 15) \\
\text { Melting temperature 1: } 123.2^{\circ} \mathrm{C} / 124.1^{\circ} \mathrm{C} / \mathrm{NA} \\
\text { Melting temperature 2: } 150.7^{\circ} \mathrm{C} / 150.7^{\circ} \mathrm{C} / 140.9^{\circ} \mathrm{C} \\
\text { Enthalpy of fusion 1:1944 J g }{ }^{-1} / 2482 \mathrm{~J} \mathrm{~g}^{-1} / \mathrm{NA} \\
\text { Enthalpy of fusion 2:1745 } \mathrm{J} \mathrm{g}^{-1} / 1745 \mathrm{~J} \mathrm{~g}^{-1} / 1509 \mathrm{~J} \mathrm{~g}^{-1} \\
\text { Glass transition temperature } 1:-28.2^{\circ} \mathrm{C} /-28.0^{\circ} \mathrm{C} /-27.4^{\circ} \mathrm{C} \\
\text { Glass transition temperature } 2: 48.6^{\circ} \mathrm{C} / 47.9^{\circ} \mathrm{C} / 36.9^{\circ} \mathrm{C} \\
\text { Crystallization temperature: } 102.4^{\circ} \mathrm{C} / 102.2^{\circ} \mathrm{C} / 99.0^{\circ} \mathrm{C} \\
\text { Chlorine loss: } 0.3 \% / 1.3 \% / 1.7 \% \\
\text { MCPA loss: } 5.1 \% / 7.4 \% / 9.7 \% \\
\mathrm{P}(3 \mathrm{HB}-\text { co-3HV) loss before bond scission: } 20.6 \% / 29.7 \% / 38.8 \% \\
\text { P(3HB-co-3HV) loss after bond scission: } 2.8 \% / 2.4 \% / 2.4 \%\end{array}$ & Mulch & [32] \\
\hline $\begin{array}{c}\frac{\mathrm{mPEG}}{\text { Incorporation method: }} \\
\text { Transesterification } \\
\text { 3HV fraction: } 12 \text { and } 33 \mathrm{~mol} \%\end{array}$ & $\begin{array}{c}\text { P(3HB-co-3HV):mPEG, } 12 \mathrm{~mol} \% / 33 \mathrm{~mol} \% 3 \mathrm{HV} \\
\text { Number average molecular weight: } 8980 / 4980 \\
\text { Weight average molecular weight: } 6200 / 2650 \\
\text { Polydispersity index: } 1.44 / 1.84 \\
\text { Melting temperature of } \mathrm{P}(3 \mathrm{HB}-\mathrm{co}-3 \mathrm{HV}) \text { block: } 140.5^{\circ} \mathrm{C} / 133.6{ }^{\circ} \mathrm{C} \\
\text { Melting temperature of mPEG block: } 49.1^{\circ} \mathrm{C} / 49.3{ }^{\circ} \mathrm{C} \\
\text { Particle size: } 162 \mathrm{~nm} / 125 \mathrm{~nm} \\
\text { Encapsulation efficiency: } 43 \% / 57 \% \\
\text { Cytotoxicity }(100 \rightarrow 500 \mu \mathrm{m} / \mathrm{mL} \text { nanoparticles }): 94 \% \rightarrow 80 \% / 88 \% \\
\rightarrow 78 \%\end{array}$ & $\begin{array}{l}\text { Drug } \\
\text { delivery } \\
\text { carrier }\end{array}$ & [24] \\
\hline
\end{tabular}


Table 1. Cont.

\begin{tabular}{|c|c|c|c|}
\hline Incorporated Components $\mathrm{A}$ & Changes in the Properties & $\begin{array}{c}\text { Potential } \\
\text { Applications }\end{array}$ & Ref. \\
\hline $\begin{array}{c}\mathrm{NH}_{2}-g \text {-collagen or } \\
\text { PHEMA-g-collagen } \\
\text { Incorporation method: } \\
\text { Solvent } \\
\text { casting } \\
\text { followed by } \\
\text { solute leaching technique } \\
\text { 3HV fraction: } 12 \mathrm{~mol} \%\end{array}$ & 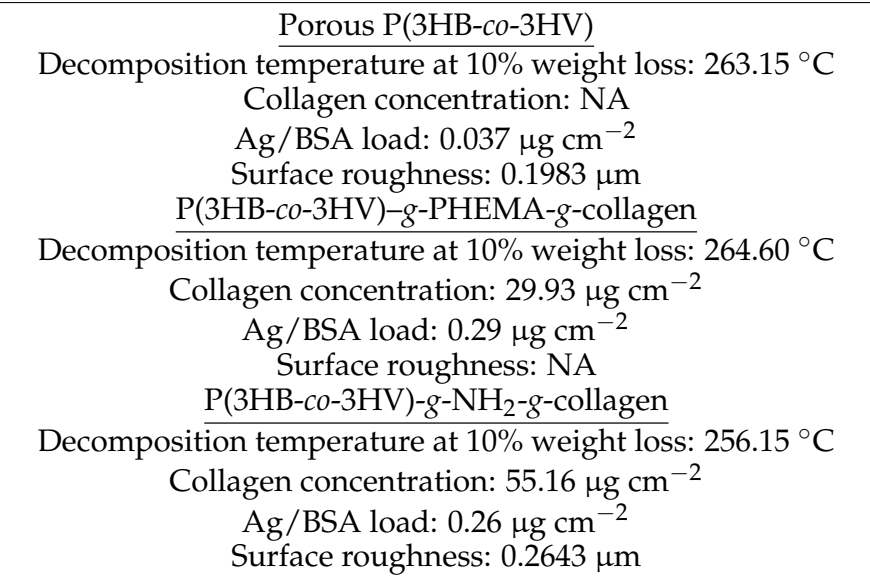 & $\begin{array}{l}\text { Bone } \\
\text { implant }\end{array}$ & [33] \\
\hline $\begin{array}{l}\text { NR } \\
\text { Incorporation method: } \\
\text { Twin screw extrusion } \\
\text { 3HV fraction: } 3 \text { mol\% }\end{array}$ & $\begin{array}{c}\mathrm{P}(3 \mathrm{HB}-\mathrm{co}-3 \mathrm{HV}) \mathrm{NR}(100: 0 / 85: 15) \\
\text { Melting temperature: } 172.05^{\circ} \mathrm{C} / 171.95^{\circ} \mathrm{C} \\
\mathrm{P}(3 \mathrm{HB}-\mathrm{co}-3 \mathrm{HV}) \text { glass transition temperature: } 5.65^{\circ} \mathrm{C} / 6.05^{\circ} \mathrm{C} \\
\text { NR glass transition temperature: NA } /-64.5^{\circ} \mathrm{C} \\
\text { Crystallization temperature: } 120.85^{\circ} \mathrm{C} / 119.45^{\circ} \mathrm{C} \\
\text { Degree of crystallinity: } 74.7 \% / 61.6 \% \\
\text { Tensile strength: } 43 \mathrm{MPa} / 26 \mathrm{MPa} \\
\text { Elongation at break: } 8 \% / 16 \% \\
\text { Notched impact strength: } 15 \mathrm{~J} \mathrm{~m}{ }^{-1} / 14 \mathrm{~J} \mathrm{~m}^{-1} \\
\text { Secant modulus: } 12 \mathrm{GPa} / 0.9 \mathrm{GPa}\end{array}$ & $\begin{array}{c}\text { Packaging } \\
\text { material }\end{array}$ & [34] \\
\hline $\begin{array}{l}\text { PBAT } \\
\text { Incorporation method: } \\
\text { Conventional injection } \\
\text { molding or microcellular } \\
\text { injection molding } \\
\text { 3HV fraction: NA }\end{array}$ & $\begin{array}{c}\text { Solid P(3HB-co-3HV):PBAT }(98.5: 1.5 \rightarrow 30: 70) \\
\text { Melting temperature: } 166.2 \rightarrow 170.4^{\circ} \mathrm{C} \\
\text { Cold crystallization temperature: } \mathrm{NA} \rightarrow 44.7^{\circ} \mathrm{C} \\
\text { Degree of crystallinity: } 78 \% \rightarrow 29 \% \\
\text { Specific toughness: } 5.3 \times 10^{-4} \rightarrow 7.1 \times 10^{-2} \mathrm{MPa} \mathrm{kg}{ }^{-1} \mathrm{~m}^{-3} \\
\text { Elongation at break: } 2.7 \% \rightarrow 555.7 \% \\
\text { Specific tensile strength: } \\
3.2 \times 10^{-2} \rightarrow 1.5 \times 10^{-2} \mathrm{MPa} \mathrm{kg}{ }^{-1} \mathrm{~m}^{-3} \\
\text { Specific Young's modulus: } 2.2 \rightarrow 0.5 \mathrm{MPa} \mathrm{kg}{ }^{-1} \mathrm{~m}^{-3} \\
\text { Microcellular P(3HB-co-3HV):PBAT }(98.5: 1.5 \rightarrow 30: 70) \\
\text { Melting temperature: } 167.1 \rightarrow 169.6^{\circ} \mathrm{C} \\
\text { Cold crystallization temperature: NA } \rightarrow 45.7^{\circ} \mathrm{C} \\
\text { Degree of crystallinity: } 80 \% \rightarrow 25 \% \\
\text { Specific toughness: } 3.8 \times 10^{-4} \rightarrow 5.8 \times 10^{-2} \mathrm{MPa} \mathrm{kg} \mathrm{m}^{-1} \mathrm{~m}^{-3} \\
\text { Elongation at break: } 2.2 \% \rightarrow 493.9 \% \\
\text { Specific tensile strength: } \\
2.7 \times 10^{-2} \rightarrow 1.3 \times 10^{-2} \mathrm{MPa} \mathrm{kg} \mathrm{m}^{-1} \mathrm{~m}^{-3} \\
\text { Specific Young's modulus: } 2.1 \rightarrow 0.5 \mathrm{MPa} \mathrm{kg}{ }^{-1} \mathrm{~m}^{-3}\end{array}$ & $\begin{array}{c}\text { Packaging } \\
\text { material }\end{array}$ & [35] \\
\hline $\begin{array}{l}\text { PBS } \\
\text { Incorporation method: } \\
\text { Solvent casting } \\
\text { 3HV fraction: } 14 \mathrm{~mol} \%\end{array}$ & $\begin{array}{c}\text { P(3HB-co-3HV):PBS }(100: 0 \rightarrow 40: 60) \\
\text { Crystallization time at } 60{ }^{\circ} \mathrm{C}: 8 \rightarrow 14.5 \mathrm{~min} \\
\text { Overall crystallization constant: } \\
3.13 \times 10^{-2} \rightarrow 2.22 \times 10^{-3} \mathrm{~min}^{-\mathrm{n}} \\
\text { Avrami index: } 2.57 \rightarrow 2.67\end{array}$ & $\begin{array}{c}\text { Packaging } \\
\text { material }\end{array}$ & [36] \\
\hline $\begin{array}{l}\text { PBS-DCP } \\
\text { Incorporation method: } \\
\text { Compression molding } \\
\text { 3HV fraction: } 13 \text { mol\% }\end{array}$ & $\begin{array}{c}\text { P(3HB-co-3HV):PBS }(100: 0 \rightarrow 70: 30) \\
\text { Tensile strength: } 22 \rightarrow 23 \mathrm{MPa} \\
\text { Elongation at break: } 4.5 \% \rightarrow 6.5 \% \\
80 \mathrm{wt} \% \mathrm{P}(3 \mathrm{HB}-\mathrm{co}-3 \mathrm{HV})-20 \mathrm{wt} \% \mathrm{PBS}: \mathrm{DCP}(100: 0 \rightarrow 99: 1) \\
\text { Tensile strength: } 25 \rightarrow 27 \mathrm{MPa} \\
\text { Elongation at break: } 8 \% \rightarrow 350 \% \\
\text { Notched Izod impact toughness: } 2.8 \rightarrow 5.5 \mathrm{~kJ} \mathrm{~m}{ }^{-2} \\
\text { Flexural strength: } 39 \rightarrow 30 \mathrm{MPa} \\
\text { Flexural modulus: } 1.2 \rightarrow 0.6 \mathrm{GPa}\end{array}$ & $\begin{array}{c}\text { Packaging } \\
\text { material }\end{array}$ & [37] \\
\hline
\end{tabular}


Table 1. Cont.

\section{Incorporated Components ${ }^{A}$}

PCL

Incorporation method: Solvent casting 3HV fraction: $7 \mathrm{~mol} \%$
Changes in the Properties

$\mathrm{P}(3 \mathrm{HB}-\mathrm{co}-3 \mathrm{HV}) / \mathrm{PCL}$

Number average molecular weight: $127,000 / 56,400$

Weight average molecular weight: $470,000 / 163,300$

Melting temperature: $151.2{ }^{\circ} \mathrm{C} / 64.0^{\circ} \mathrm{C}$

Glass transition temperature: $5.2{ }^{\circ} \mathrm{C} /-61.0^{\circ} \mathrm{C}$

Crystallization temperature: $97.0^{\circ} \mathrm{C} / 22.2^{\circ} \mathrm{C}$ $\mathrm{P}(3 \mathrm{HB}-\mathrm{co}-3 \mathrm{HV}): \mathrm{PCL}(100: 0 \rightarrow 50: 50)$

Isothermal crystallization temperature: $120 \rightarrow 120^{\circ} \mathrm{C}$

Overall crystallization constant: $2.20 \times 10^{-7} \rightarrow 1.00 \times 10^{-8} \mathrm{~s}^{-\mathrm{n}}$

Avrami index: $2.80 \rightarrow 2.66$

Potential

Applications

Ref.

Packaging material

P(3HB-co-3HV):PDLLA (100:0 $\rightarrow$ 30:70)

Melting temperature: $157.8 \rightarrow 169.8^{\circ} \mathrm{C}$

Degree of crystallinity: $53.6 \rightarrow 9.9^{\circ} \mathrm{C}$

Tensile strength: $19.7 \rightarrow 49.7 \mathrm{MPa}$

Elongation at break: $0.17 \% \rightarrow 2.07 \%$

Flexural strength: $39.1 \rightarrow 75.0 \mathrm{MPa}$

Flexural modulus:3646 $\rightarrow 3507 \mathrm{MPa}$

PDLLA-PEG

Incorporation method:

Compression molding

3HV fraction: $1 \mathrm{~mol} \%$
Burial biodegradation (day 30 ): $0 \% \rightarrow 1 \%$

$30 \mathrm{wt} \% \mathrm{P}(3 \mathrm{HB}-c o-3 \mathrm{HV})-70 \mathrm{wt} \%$ PDLLA:PEG $(90: 10 \rightarrow 80: 20)$

Melting temperature: $171.2 \rightarrow 170.8^{\circ} \mathrm{C}$

Degree of crystallinity: $10.5 \rightarrow 13.0^{\circ} \mathrm{C}$

Tensile strength: $29.7 \rightarrow 24.1 \mathrm{MPa}$

Elongation at break: $28.7 \% \rightarrow 237.0 \%$

Flexural strength: $36.1 \rightarrow 5.48 \mathrm{MPa}$

Flexural modulus: $1127 \rightarrow 220 \mathrm{MPa}$

Burial biodegradation (day 30 ): $3 \% \rightarrow 11 \%$

\section{PEG}

Incorporation method: Solvent casting

3HV fraction: $4 \mathrm{~mol} \%$

PEG

Incorporation method:

Solvent casting

3HV fraction: NA

P(3HB-co-3HV):PEG $(100: 0 \rightarrow 20: 80)$
Melting temperature: $163.2 \rightarrow 145.0^{\circ} \mathrm{C}$
Enthalpy of fusion: $89.62 \rightarrow 1.63 \mathrm{~J} \mathrm{~g}^{-1}$

$$
\mathrm{P}(3 \mathrm{HB}-\mathrm{co}-3 \mathrm{HV})
$$

Melting temperature: $90^{\circ} \mathrm{C}$

Initial thermal degradation temperature: $220^{\circ} \mathrm{C}$

Final thermal degradation temperature: $255^{\circ} \mathrm{C}$

Tensile strength: $10.3 \mathrm{MPa}$

Elongation at break: $13.3 \%$ Cytotoxicity: $20 \%$

P(3HB-co-3HV):PEG (4:1)

Cytotoxicity: $0 \%-10 \%$

\section{$\mathrm{P}(3 \mathrm{HB}-\mathrm{co}-3 \mathrm{HV}) / \mathrm{PLA}$}

Melting temperature: $172{ }^{\circ} \mathrm{C} / 170{ }^{\circ} \mathrm{C}$

Glass transition temperature: $5^{\circ} \mathrm{C} / 64^{\circ} \mathrm{C}$

Enthalpy of fusion: $92.8 \mathrm{~J} \mathrm{~g}^{-1} / 44.2 \mathrm{~J} \mathrm{~g}^{-1}$

Crystallization temperature: $122^{\circ} \mathrm{C} / 112{ }^{\circ} \mathrm{C}$

Decomposition temperature: $303^{\circ} \mathrm{C} / 382{ }^{\circ} \mathrm{C}$

Izod impact strength: $1.99 \mathrm{~kJ} \mathrm{~m}^{-2} / 2.14 \mathrm{~kJ} \mathrm{~m}^{-2}$

Flexural strength: $47.70 \mathrm{MPa} / 58.07 \mathrm{MPa}$

Flexural modulus: $3.48 \mathrm{GPa} / 2.94 \mathrm{GPa}$

PLA-CNT

Incorporation method: High-speed spinning 3HV fraction: $2 \mathrm{~mol} \%$
$80 \mathrm{wt} \% \mathrm{P}(3 \mathrm{HB}-\mathrm{co}-3 \mathrm{HV})-20 \mathrm{wt} \% \mathrm{PLA}: \mathrm{CNT}(100: 0 \rightarrow 99: 1)$

Melting temperature: $169 \rightarrow 168^{\circ} \mathrm{C}$

Glass transition temperature: $-2 \rightarrow-2{ }^{\circ} \mathrm{C}$

Enthalpy of fusion: $44.11 \rightarrow 48.10 \mathrm{~J} \mathrm{~g}^{-1}$

Crystallization temperature: $112 \rightarrow 122{ }^{\circ} \mathrm{C}$

Decomposition temperature: $379 \rightarrow 380^{\circ} \mathrm{C}$

Izod impact strength: $4.10 \rightarrow 2.46 \mathrm{~kJ} \mathrm{~m}^{-2}$

Flexural strength: $51.60 \rightarrow 61.01 \mathrm{MPa}$

Flexural modulus: $3.10 \rightarrow 3.25 \mathrm{GPa}$

Electrical conductivity: $8.67 \times 10^{-14} \rightarrow 2.79 \times 10^{-2} \mathrm{~S} \mathrm{~m}^{-1}$

Reflectivity (frequency): $0 \mathrm{~dB}(\mathrm{NA}) \rightarrow-15 \mathrm{~dB}(11 \mathrm{GHz})$
Biomedical, agricultural and packaging material

Drug

delivery

carrier

Skin grafting

Electrical and electromagnetic 
Table 1. Cont.

\begin{tabular}{|c|c|c|c|}
\hline Incorporated Components ${ }^{A}$ & Changes in the Properties & $\begin{array}{c}\text { Potential } \\
\text { Applications }\end{array}$ & Ref. \\
\hline $\begin{array}{l}\text { PLA-nanoclay } \\
\text { Incorporation method: } \\
\text { Twin screw extrusion } \\
\text { 3HV fraction: NA }\end{array}$ & $\begin{array}{c}\text { P(3HB-co-3HV):PLA }(15: 85 \rightarrow 30: 70) \\
\text { Melting temperature: } 154.75 \rightarrow 156.40{ }^{\circ} \mathrm{C} \\
\text { Cold crystallization temperature: } 133.45 \rightarrow 121.89{ }^{\circ} \mathrm{C} \\
\text { Degree of crystallinity: } 1.98 \% \rightarrow 4.33 \% \\
\text { Tensile strength: } 52.5 \rightarrow 47.5 \mathrm{MPa} \\
\text { Elongation at break: } 9.0 \% \rightarrow 6.0 \% \\
\text { Young's modulus: } 1700 \rightarrow 1750 \mathrm{MPa} \\
\text { P(3HB-co-3HV)-PLA:nanoclay }(15: 85 \rightarrow 30: 70) \\
\text { Melting temperature: } 156.52 \rightarrow 157.43{ }^{\circ} \mathrm{C} \\
\text { Cold crystallization temperature: } 129.09 \rightarrow 111.04{ }^{\circ} \mathrm{C} \\
\text { Degree of crystallinity: } 13.05 \% \rightarrow 18.40 \% \\
\text { Tensile strength: } 49.2 \rightarrow 48.0 \mathrm{MPa} \\
\text { Elongation at break: } 8.5 \% \rightarrow 4.0 \% \\
\text { Young's modulus: } 2060 \rightarrow 2060 \mathrm{MPa}\end{array}$ & $\begin{array}{c}\text { Packaging } \\
\text { material }\end{array}$ & {$[42]$} \\
\hline $\begin{array}{l}\text { PPC } \\
\text { Incorporation method: } \\
\text { Solvent casting } \\
\text { 3HV fraction: } 5 \mathrm{~mol} \%\end{array}$ & $\begin{array}{l}\qquad \begin{array}{l}\text { P(3HB-co-3HV):PPC }(100: 0 \rightarrow 20: 80) \\
\text { Melting temperature: } 163 \rightarrow 162{ }^{\circ} \mathrm{C}\end{array} \\
\text { Thermal decomposition temperature: } 199 \rightarrow 190^{\circ} \mathrm{C} \\
\text { Maximum mass loss rate temperature: } 286 \rightarrow 267^{\circ} \mathrm{C} \\
\text { Burial biodegradation: } 100 \% \text { (day } 12) \rightarrow 85 \% \text { (day 30) }\end{array}$ & $\begin{array}{c}\text { Packaging } \\
\text { material }\end{array}$ & [43] \\
\hline $\begin{array}{l}\text { starch, cellulose or alginate } \\
\text { Incorporation method: } \\
\text { Solvent casting } \\
\text { 3HV fraction: } 6 \mathrm{~mol} \%\end{array}$ & $\begin{array}{c}\text { P(3HB-co-3HV)-starch }(100: 0 \rightarrow 30: 70) \\
\text { Tensile strength: } 25 \rightarrow 1 \mathrm{MPa} \\
\text { Elongation at break: } 8 \% \rightarrow 4 \% \\
\text { Young's modulus: } 181 \rightarrow 4 \mathrm{MPa} \\
\text { Density: } 0.974 \rightarrow 1.243 \mathrm{~g} \mathrm{~cm}^{-3} \\
\text { Solubility: } 0 \% \rightarrow 6.0 \% \\
\text { Water absorption capacity: } 0 \% \rightarrow 21.0 \% \\
\text { Burial biodegradation (day } 30): 10 \% \rightarrow 100 \% \\
\text { Immersion biodegradation (day } 30): 23 \% \rightarrow 100 \% \\
\text { P(3HB-co-3HV)-cellulose }(100: 0 \rightarrow 30: 70) \\
\text { Tensile strength: } 25 \rightarrow 1 \mathrm{MPa} \\
\text { Elongation at break: } 8 \% \rightarrow 3 \% \\
\text { Young's modulus: } 181 \rightarrow 7 \mathrm{MPa} \\
\text { Density: } 0.974 \rightarrow 1.212 \mathrm{~g} \mathrm{~cm}-3 \\
\text { Solubility: } 0 \% \rightarrow 1.7 \% \\
\text { Water absorption capacity: } 0 \% \rightarrow 4.7 \% \\
\text { Burial biodegradation }(\text { day } 30): 10 \% \rightarrow 70 \% \\
\text { Immersion biodegradation }(\text { day } 30): 23 \% \rightarrow 100 \% \\
\text { P(3HB-co-3HV)-arginate }(100: 0 \rightarrow 30: 70) \\
\text { Tensile strength: } 25 \rightarrow 1 \mathrm{MPa} \\
\text { Elongation at break: } 8 \% \rightarrow 2 \% \\
\text { Young's modulus: } 181 \rightarrow 3 \mathrm{MPa} \\
\text { Density: } 0.974 \rightarrow 1.053 \mathrm{~g} \mathrm{~cm}-3 \\
\text { Solubility: } 0 \% \rightarrow 19.0 \% \\
\text { Water absorption capacity: } 0 \% \rightarrow 33.0 \% \\
\text { Burial biodegradation }(\text { day } 30): 10 \% \rightarrow 80 \% \\
\text { Immersion biodegradation }(\text { day } 30): 21 \% \rightarrow 100 \%\end{array}$ & Mulch & [44] \\
\hline
\end{tabular}


Table 1. Cont.

\begin{tabular}{|c|c|c|c|}
\hline Incorporated Components ${ }^{A}$ & Changes in the Properties & $\begin{array}{c}\text { Potential } \\
\text { Applications }\end{array}$ & Ref. \\
\hline $\begin{array}{c}\text { ZnO } \\
\text { Incorporation method: } \\
\text { Melt-mixed compression } \\
\text { molding, electrospinning or } \\
\text { coating } \\
\text { 3HV fraction: } 3 \text { and } 18 \mathrm{~mol} \%\end{array}$ & $\begin{array}{c}\mathrm{P}(3 \mathrm{HB}-\mathrm{co}-3 \mathrm{~mol} \% 3 \mathrm{HV}) / \mathrm{P}(3 \mathrm{HB}-\mathrm{co}-18 \mathrm{~mol} \% 3 \mathrm{HV}) \\
\text { Melting temperature: } 168.7^{\circ} \mathrm{C} / 170.9^{\circ} \mathrm{C} \\
\text { Decomposition temperature: } 290.8^{\circ} \mathrm{C} / 283.1{ }^{\circ} \mathrm{C} \\
\text { Crystallization temperature: } 114.7^{\circ} \mathrm{C} / 101.0^{\circ} \mathrm{C} \\
\text { Degree of crystallinity: } 66 \% / 63 \% \\
\text { Tensile strength: } 33.9 \mathrm{MPa} / 18.5 \mathrm{MPa} \\
\text { Elongation at break: } 1.5 \% / 1.3 \% \\
\text { Young's modulus: } 2.6 \mathrm{GPa} / 2.2 \mathrm{GPa} \\
\mathrm{L}^{*}, \mathrm{a}^{*}, \mathrm{~b}^{*}: 82.3,1.4,17.7 / 32.7,6.7,10.2 \\
\text { PHBVs-D/PHBVs-P/PHBVs-C } \mathrm{B} \\
\text { Melting temperature: } 166.9^{\circ} \mathrm{C} / 166.5^{\circ} \mathrm{C} / 169.0^{\circ} \mathrm{C} \\
\text { Decomposition temperature: } 271.3^{\circ} \mathrm{C} / 270.3^{\circ} \mathrm{C} / 270.8^{\circ} \mathrm{C} \\
\text { Crystallization temperature: } 112.1^{\circ} \mathrm{C} / 111.6^{\circ} \mathrm{C} / 118.0^{\circ} \mathrm{C} \\
\text { Degree of crystallinity: } 50 \% / 51 \% / 35 \% \\
\text { Tensile strength: } 12.5 \mathrm{MPa} / 34.8 \mathrm{MPa} / 22.6 \mathrm{MPa} \\
\text { Elongation at break: } 6.5 \% / 2.3 \% / 6.2 \% \\
\text { Young's modulus: } 1.5 \mathrm{GPa} / 2.1 \mathrm{GPa} / 1.4 \mathrm{GPa} \\
\mathrm{L}^{*}, \mathrm{a}^{*}, \mathrm{~b}^{*}: 56.9,9.0,25.3 / 58.4,8.5,25.1 / 72.5,3.8,24.7\end{array}$ & $\begin{array}{l}\text { Active food } \\
\text { packaging } \\
\text { and } \\
\text { food } \\
\text { contact } \\
\text { surface } \\
\text { applications }\end{array}$ & [45] \\
\hline $\begin{array}{l}\frac{\mathrm{ZnO}}{\text { Incorporation method: }} \\
\text { Laser 3D molding } \\
\text { 3HV fraction: NA }\end{array}$ & $\begin{array}{c}\text { P(3HB-co-3HV)-ZnO }(100: 0 \rightarrow 95: 5) \\
\text { Melting temperature: } 171 \rightarrow 158^{\circ} \mathrm{C} \\
\text { Decomposition temperature: } 261.2 \rightarrow 288.7^{\circ} \mathrm{C} \\
\text { Strain:14.0\% } \rightarrow 9.5 \% \\
\text { Stress: } 3.5 \rightarrow 4.5 \mathrm{MPa} \\
\text { Compression strength: } 4 \rightarrow 5 \mathrm{MPa} \\
\text { Compression modulus: } 60 \rightarrow 80 \mathrm{MPa} \\
\text { Bacterial inhibition rate (day } 5): 2.5 \% \rightarrow 79.0 \% \\
\left.\mathrm{Zn}^{2+} \text { release in deionized water (day } 7\right): 0.19 \rightarrow 0.34 \mathrm{mg} \mathrm{L}^{-1}\end{array}$ & $\begin{array}{l}\text { Bone } \\
\text { repair }\end{array}$ & [46] \\
\hline
\end{tabular}

A Synthetic atactic poly(3-hydroxybutyrate) $(\alpha-\mathrm{P}(3 \mathrm{HB}))$, bovine serum albumin capped silver (Ag/BSA), ascorbic acid (AS), cellulose nanocrystals (CNC), carbon nanotubes (CNT), dicumyl peroxide (DCP), distillers' dried grains with solubles (DDGS), hydroxyapatite (HA), organophilic attapulgite (MAT), Miscanthus (Misc), 2methyl-4-chlorophenoxyacetic acid (MCPA), monomethoxy poly(ethylene glycol) (mPEG), natural rubber (NR), poly( $\varepsilon$-caprolactone) (PCL), poly(d,l-lactide) (PDLLA), poly(butylene succinate) (PBS), poly(butylene adipate-coterephthalate) (PBAT), poly(ethylene glycol) (PEG), poly(2-hydroxyl ethyl methacrylate) (PHEMA), poly(lactic acid) (PLA), poly(propylene carbonate) (PPC), not available (NA). ${ }^{\mathrm{B}}$ Melt-mixed compress molded P(3HBco-3 mol\%3HV):P(3HB-co-18 mol\%3HV):ZnO (70:24:6) (PHBVs-D), electrospun $\mathrm{P}(3 \mathrm{HB}-\mathrm{co}-18 \mathrm{~mol} \% 3 \mathrm{HV}): \mathrm{ZnO}$ (50:50) (PHBVs-P), P(3HB-co-18 mol\%3HV):ZnO (50:50) coating on compressed molded $\mathrm{P}(3 \mathrm{HB}-\mathrm{co}-3 \mathrm{~mol} \% 3 \mathrm{HV})$ (PHBVs-C).

$\mathrm{P}(3 \mathrm{HB}-\mathrm{co}-3 \mathrm{HV})$ is a potential substitute for petroleum-based plastic packaging material as it possesses high water and aroma (limonene and linalool) barrier properties while having comparable thermal and mechanical properties to that of polypropylene (PP) and lowdensity polyethylene (LDPE) [15]. As PP and LDPE are applied extensively for packaging and consumables, which are highly disposable, the substitution with $\mathrm{P}(3 \mathrm{HB}-\mathrm{co}-3 \mathrm{HV})$ can contribute to reduced stable solid waste creation of petroleum-based plastics [47,48]. Unlike the augmented cytotoxicity by higher $3 \mathrm{HV}$ molar fraction, lower $3 \mathrm{HV}$ molar fraction causes high stereoregularity, slow crystallization rate, formation of large size spherulites, and secondary crystallization that are discouraging for packaging purposes $[24,47,48]$. Poly(butylene succinate), poly(butylene adipate-co-terephthalate), natural rubber, or other polymers with plasticizer or toughness properties can be incorporated to overcome the limitations and extend its application as packaging materials (Table 1).

Moreover, PHA-based mulch films are potential substitutes for conventional plastic mulch films. Mulching increases crops productivity, increases horticulture products, prevents water evaporation from the soil, prevents soil erosion, reduces water consumption, and controls weeds [49]. PHA-based mulch films overcome the environmental problems caused by the post-consumption of plastic mulch films made from LDPE, linear low-density polyethylene (LLDPE), and high-density polyethylene (HDPE) due to their poor degradability [50]. Moreover, the physicochemical properties of $\mathrm{P}(3 \mathrm{HB}-\mathrm{co}-3 \mathrm{HV})$ enable the controlled release of herbicides and insecticides. Herbicides and insecticides can be integrated into 
$\mathrm{P}(3 \mathrm{HB}-\mathrm{co}-3 \mathrm{HV})$-containing pellets and sown along the plantation to be released upon degradation from the pellets depending on the level of pest activity [51,52].

On the other hand, endogenous $\mathrm{P}(3 \mathrm{HB}-\mathrm{co}-3 \mathrm{HV})$ acts as the electron donor for the denitrification of wastewater in the aquaculture industry. Biomass with PHA-accumulating ability, generally $\mathrm{P}(3 \mathrm{HB})$ and poly(3-hydroxyvalerate) $(\mathrm{P}(3 \mathrm{HV}))$, from activated sludge, is employed to remove resulting ammonia from fish excretion and dead animal bodies in circulating water. Unlike the conventional techniques that involve the addition of acetate and ethanol to promote microbial activity, the biomass is precultured for PHA accumulation. The endogenous PHA is used for denitrification that accurately couples with slow metabolic activity in the absence of exogenous carbon source and in the presence of nitrogen $[53,54]$. The exclusion of volatile fatty acids feeding during the denitrification process prevents the contamination with the dissolved organic carbon that lowers the effluent water quality, and the employment of endogenous PHA is more cost-effective compared to feeding extracted PHA to denitrifying bacteria [55].

\section{Bioconversion of Alkyl Alcohols and Organic Acids into P(3HB-co-3HV)}

The conversion of organic acid into $3 \mathrm{HV}$ starts with $\beta$-oxidation, where propionic acid (C3) is converted into propionyl-CoA, whereas valeric acid (C5) is converted into propionyl$\mathrm{CoA}$ and acetyl-CoA, respectively [56]. The $3 \mathrm{HV}$ monomer is formed from the resulting propionyl-CoA couples with acetyl-CoA and is polymerized to $\mathrm{P}(3 \mathrm{HB}-\mathrm{co}-3 \mathrm{HV})$ copolymer with the $3 \mathrm{HB}$ monomer. The $3 \mathrm{HB}$ monomer is formed from the resulting acetyl-CoA provided majorly by the main carbon source such as oils or sugars (Figure 2) [14,57-60].

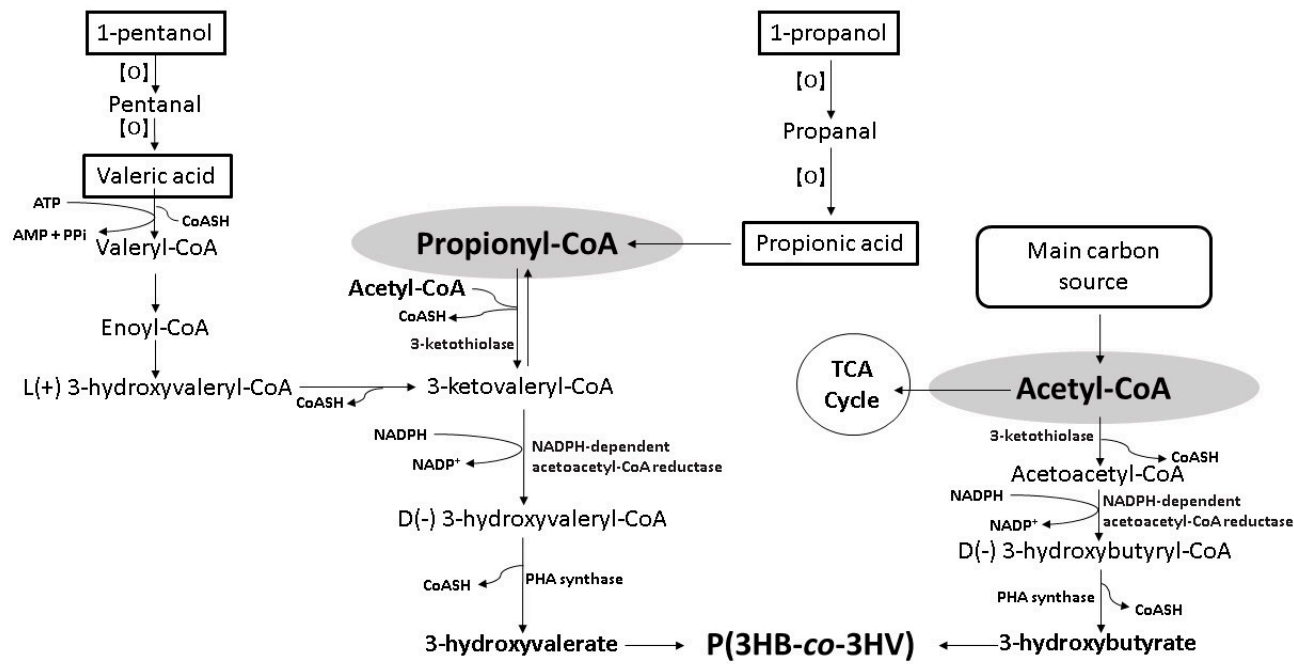

Figure 2. Schematic bioconversion pathway of organic acids and alkyl alcohols into 3HV [14,57-60].

The employment of alkyl alcohols as $3 \mathrm{HV}$ precursors is limited to odd carbon number primary alcohols. Primary alcohols are oxidized to aldehydes that can be further oxidized more easily to their respective carboxylic acids. The oxidation processes can occur chemically with the presence of oxidizing agents or biologically with the presence of alcohol dehydrogenase and aldehyde dehydrogenase [61]. Oxidation of secondary alcohols liberates ketones with no further oxidation due to the oxidatively stable nature of ketones [62,63]. Odd carbon number primary alcohols such as 1-propanol or 1-pentanol are oxidized to 1-propanal and 1-pentanal that further oxidized to propanoic acid and valeric acid, respectively. The resulting propionic acid or valeric acid enters $\beta$-oxidation to liberate propionyl-CoA for $3 \mathrm{HV}$ formation (Figure 2) [14,57-60].

Although levulinic acid is a cost-effective $3 \mathrm{HV}$ precursor, the catabolic pathway involved is undetermined. Generally, levulinic acid catabolism releases intermediates that are converted via $\beta$-oxidation to release acetyl-CoA and propionyl-CoA for $\mathrm{P}(3 \mathrm{HB}-\mathrm{co}-3 \mathrm{HV})$ biosynthesis [64]. Bacteria capable of using levulinic acid as the $3 \mathrm{HV}$ precursor are rare 
and are mainly C. necator, with the exception of Burkholderis sp. IS-01 and Hydrogenophaga pseudoflava DSM 1034 [10,11,65-68]. C. necator KHB-8862 and H. pseudoflava DSM 1034 are two promising strains reported with a high $3 \mathrm{HV}$ yield of 0.50 and $1.00 \mathrm{~g} / \mathrm{g}$, respectively. However, other studies reported low PHA content and $3 \mathrm{HV}$ yield (Table 2).

Table 2. $\mathrm{P}(3 \mathrm{HB}-\mathrm{co}-3 \mathrm{HV})$ production by bacteria from various $3 \mathrm{HV}$ precursors.

\begin{tabular}{|c|c|c|c|c|c|c|c|}
\hline \multirow{2}{*}{$\begin{array}{l}\text { Microorganisms and } \\
\text { Carbon Sources }\end{array}$} & \multirow{2}{*}{$\underset{(g / L)}{\text { Biomass }}$} & \multicolumn{2}{|c|}{ PHA Content } & \multicolumn{2}{|c|}{ 3HV Composition } & \multirow{2}{*}{$\begin{array}{c}\text { 3HV Yield } \\
\text { (g/g) }\end{array}$} & \multirow{2}{*}{ Ref. } \\
\hline & & $(w t \%)$ & $(g / L)$ & $(\mathrm{mol} \%)$ & $(g / L)$ & & \\
\hline \multicolumn{8}{|c|}{ Organic acids } \\
\hline $\begin{array}{l}\text { Bacillus aryabhattai PHB10 } \\
\text { Glucose }(20.0 \mathrm{~g} / \mathrm{L}) \\
\text { Propionic acid }(0.7 \mathrm{~g} / \mathrm{L})\end{array}$ & 3.9 & 72 & 2.8 & - & - & - & [28] \\
\hline $\begin{array}{l}\text { Bacillus thuringiensis R-510 } \\
\text { Glucose }(23.5 \mathrm{~g} / \mathrm{L}) \\
\text { Propionic acid }(1.0 \mathrm{~g} / \mathrm{L})\end{array}$ & 2.9 & 21 & 0.6 & 41 & 0.2 & 0.25 & [69] \\
\hline $\begin{array}{c}\text { C. necator DSM } 545 \\
\text { Waste glycerol }(20.0 \mathrm{~g} / \mathrm{L}) \\
\text { Propionic acid }(4.0 \mathrm{~g} / \mathrm{L})\end{array}$ & 4.5 & 57 & 2.6 & 25 & 0.7 & 0.16 & [70] \\
\hline $\begin{array}{c}\text { C. necator DSM } 545 \\
\text { Butyric acid }(246.0 \mathrm{~g} / \mathrm{L}) \\
\text { Propionic acid }(186.0 \mathrm{~g} / \mathrm{L})\end{array}$ & 65.9 & 88 & 58 & 36 & 20.8 & 0.11 & [71] \\
\hline $\begin{array}{l}\text { C. necator NRRL B } 14690 \\
\text { Fructose }(40.0 \mathrm{~g} / \mathrm{L}) \\
\text { Propionic acid }(4.0 \mathrm{~g} / \mathrm{L})\end{array}$ & 8.2 & 73 & 6.0 & 23 & 1.4 & 0.35 & [72] \\
\hline $\begin{array}{c}\text { C. necator NCIMB } 11599 \\
\text { Glucose (maintained at } 10.0-20.0 \mathrm{~g} / \mathrm{L}) \\
\text { Propionic acid }(0.52 \mathrm{~mol} / \mathrm{mol} \text { glucose) }\end{array}$ & 112.3 & 57 & 64.0 & 14 & 15.7 & - & [73] \\
\hline $\begin{array}{l}\text { Erwinia sp. USMI-20 } \\
\text { Palm oil }(4.6 \mathrm{~g} / \mathrm{L}) \\
\text { Propionic acid }(1.9 \mathrm{~g} / \mathrm{L})\end{array}$ & 4.2 & 40 & 1.7 & 34 & 0.6 & 0.30 & {$[60]$} \\
\hline $\begin{array}{c}\text { Activated sludge mixed culture } \\
\text { Acetic acid, lactic acid, propionic acid }\end{array}$ & - & - & - & $31-66$ & - & - & [74] \\
\hline $\begin{array}{l}\text { Bacillus cereus RCL } 02 \\
\text { Glucose }(25.0 \mathrm{~g} / \mathrm{L}) \\
\text { Valeric acid }(1.9 \mathrm{~g} / \mathrm{L})\end{array}$ & 8.1 & 72 & 5.8 & 15 & 0.9 & 0.46 & [75] \\
\hline $\begin{array}{l}\text { C. malaysiensis USMAA9-39 } \\
\text { Oleic acid }(6.5 \mathrm{~g} / \mathrm{L}) \\
\text { Valeric acid }(0.9 \mathrm{~g} / \mathrm{L})\end{array}$ & 5.2 & 43 & 2.2 & 17 & 0.4 & 0.42 & [76] \\
\hline $\begin{array}{c}\text { C. necator DSM } 545 \\
\text { Waste glycerol }(20.0 \mathrm{~g} / \mathrm{L}) \\
\text { Valeric acid }(4.0 \mathrm{~g} / \mathrm{L})\end{array}$ & 5.3 & 64 & 3.4 & 31 & 1.1 & 0.26 & [70] \\
\hline $\begin{array}{l}\text { C. necator NRRL B } 14690 \\
\text { Fructose }(40.0 \mathrm{~g} / \mathrm{L}) \\
\text { Valeric acid }(4.0 \mathrm{~g} / \mathrm{L})\end{array}$ & 7.2 & 40 & 2.9 & 62 & 1.8 & 0.45 & [72] \\
\hline $\begin{array}{l}\text { Erwinia sp. USMI-20 } \\
\text { Palm oil }(4.6 \mathrm{~g} / \mathrm{L}) \\
\text { Valeric acid }(2.0 \mathrm{~g} / \mathrm{L})\end{array}$ & 4.8 & 34 & 1.6 & 47 & 0.3 & 0.14 & [60] \\
\hline $\begin{array}{c}\text { Methylobacterium organophilum NCIB } 11278 \\
\text { Methanol }(4.0 \mathrm{~g} / \mathrm{L}) \\
\text { Valeric aid }(0.5 \mathrm{~g} / \mathrm{L})\end{array}$ & 2.5 & 50 & 1.3 & 10 & 0.1 & 0.25 & [77] \\
\hline $\begin{array}{l}\text { Burkholderis sp. IS-01 } \\
\text { Gluconate }(20.0 \mathrm{~g} / \mathrm{L}) \\
\text { Levulinic acid }(12.5 \mathrm{~g} / \mathrm{L})\end{array}$ & 5.9 & 62 & 3.7 & 87 & 3.2 & 0.25 & [67] \\
\hline $\begin{array}{c}\text { C. necator KHB-8862 } \\
\text { Fructose syrup }(20.0 \mathrm{~g} / \mathrm{L}) \\
\text { Levulinic acid } \\
(1.0 \mathrm{~g} / \mathrm{L}, \text { initial and } 3 \text { times feeding })\end{array}$ & 8.6 & 84 & 7.2 & 28 & 2.0 & 0.50 & [66] \\
\hline
\end{tabular}


Table 2. Cont.

\begin{tabular}{|c|c|c|c|c|c|c|c|}
\hline \multirow{2}{*}{$\begin{array}{l}\text { Microorganisms and } \\
\text { Carbon Sources }\end{array}$} & \multirow{2}{*}{$\begin{array}{c}\text { Biomass } \\
(\mathrm{g} / \mathrm{L})\end{array}$} & \multicolumn{2}{|c|}{ PHA Content } & \multicolumn{2}{|c|}{ 3HV Composition } & \multirow{2}{*}{$\underset{(\mathrm{g} / \mathrm{g})}{3 \mathrm{HV} \text { Yield }}$} & \multirow{2}{*}{ Ref. } \\
\hline & & $(w t \%)$ & $(g / L)$ & $(\mathrm{mol} \%)$ & $(g / L)$ & & \\
\hline $\begin{array}{c}\text { C. necator } \mathrm{H} 16 \\
\text { Fructose }(20.0 \mathrm{~g} / \mathrm{L}) \\
\text { Levulinic acid }(3.5 \mathrm{~g} / \mathrm{L})\end{array}$ & 7.3 & 48 & 3.5 & 16 & 0.6 & 0.16 & [11] \\
\hline $\begin{array}{c}\text { Hydrogenophaga pseudoflava DSM } 1034 \\
\text { Whey permeate }(47 \mathrm{~mL} / \mathrm{L}) \\
\text { Levulinic acid }(1.0 \mathrm{~g} / \mathrm{L})\end{array}$ & 4.5 & 49 & 2.2 & 45 & 1.0 & 1.00 & [68] \\
\hline \multicolumn{8}{|c|}{ Conjugate bases of organic acids } \\
\hline $\begin{array}{l}\text { Caldimonas taiwanensis } \\
\text { Sugars }(1.5 \%) \\
\text { Valerate }(0.5 \mathrm{~g} / \mathrm{L})\end{array}$ & $1.6-4.1$ & $42-67$ & $0.8-2.1$ & $10-13$ & $0.1-0.2$ & $0.16-0.49$ & [78] \\
\hline $\begin{array}{c}\text { Methylocystis dominated mixed culture } \\
\text { Methane gas } \\
\text { (repeating } 48 \mathrm{~h} \text { fed-batch cycle) } \\
\text { Valerate }(0.4 \mathrm{~g} / \mathrm{L})\end{array}$ & 1.5 & 30 & 0.5 & 39 & 0.2 & 0.45 & [79] \\
\hline \multicolumn{8}{|c|}{ Sodium salts of organic acids } \\
\hline $\begin{array}{l}\text { Azohydromonas lata } \\
\text { Rice wastewater }(21 \mathrm{~g} / \mathrm{L}) \\
\text { Sodium acetate }(10 \mathrm{~g} / \mathrm{L})\end{array}$ & 5.0 & 32 & 1.6 & 6 & 0.1 & 0.01 & {$[80]$} \\
\hline $\begin{array}{c}\text { Corynebacterium glutamicum ATCC13869 } \\
\text { transformant } \mathrm{A} \\
\text { Sodium propionate }(1.0 \mathrm{~g} / \mathrm{L})\end{array}$ & - & 31 & - & 28 & - & - & [81] \\
\hline $\begin{array}{c}\text { C. necator } \mathrm{H} 16 \\
\text { Sodium acetate }(0-20 \mathrm{~g} / \mathrm{L}) \\
\text { Sodium propionate }(0-20 \mathrm{~g} / \mathrm{L})\end{array}$ & $0.3-0.7$ & $12-56$ & Trace & $0-45$ & Trace & - & [82] \\
\hline $\begin{array}{c}\text { C. necator } \mathrm{PHB}^{-} 4_{4}^{\mathrm{C}} \\
\text { Palm kernel oil }(5.0 \mathrm{~g} / \mathrm{L}) \\
\text { Sodium propionate }(5.0 \mathrm{~g} / \mathrm{L})\end{array}$ & 3.0 & 30 & 0.9 & 12 & 0.1 & 0.02 & [83] \\
\hline $\begin{array}{c}\text { Herbaspirillum seropedicae Z69Prp }{ }^{\mathrm{D}} \\
\text { Glucose }(7.0 \mathrm{~g} / \mathrm{L}) \\
\text { Sodium propionate }(0.5 \mathrm{~g} / \mathrm{L})\end{array}$ & 2.4 & 37 & 0.9 & 14 & 0.1 & 0.25 & [84] \\
\hline $\begin{array}{c}\text { C. necator } \mathrm{H} 16 \\
\text { Plant oils }(5.0 \mathrm{~g} / \mathrm{L}) \\
\text { Sodium valerate }(5.0 \mathrm{~g} / \mathrm{L})\end{array}$ & $4.1-6.1$ & $64-89$ & $2.1-5.4$ & $3-14$ & $0.1-0.9$ & $0.03-0.17$ & [85] \\
\hline $\begin{array}{c}\text { C. necator } \mathrm{PHB}^{-}{ }_{4} \mathrm{C} \\
\text { Palm kernel oil }(5.0 \mathrm{~g} / \mathrm{L}) \\
\text { Sodium valerate }(1.0 \mathrm{~g} / \mathrm{L})\end{array}$ & 4.2 & 52 & 2.2 & 6 & 0.1 & 0.13 & [83] \\
\hline $\begin{array}{l}\text { Methylocystis parvus OBB3 } \\
\text { Methane gas }(75 \mathrm{~mL}) \\
\text { Sodium valerate }(1.0 \mathrm{~g} / \mathrm{L})\end{array}$ & - & - & 0.3 & - & 0.2 & 0.20 & [86] \\
\hline \multicolumn{8}{|c|}{ Alkyl alcohols } \\
\hline $\begin{array}{c}\text { C. necator H16 } \\
\text { Waste rapeseed oil }(20.0 \mathrm{~g} / \mathrm{L}) \\
\text { 1-propanol }(8.0 \mathrm{~g} / \mathrm{L})\end{array}$ & 14.7 & 80 & 11.7 & 9 & 1.1 & 0.14 & [13] \\
\hline $\begin{array}{l}\text { Erwinia sp. USMI-20 } \\
\text { Palm oil }(4.6 \mathrm{~g} / \mathrm{L}) \\
\text { 1-propanol }(2.3 \mathrm{~g} / \mathrm{L})\end{array}$ & 5.4 & 50 & 2.7 & 6 & 0.2 & 0.07 & [60] \\
\hline $\begin{array}{l}\text { C. malaysiensis USMAA2-4 } \\
\text { Oleic acid }(6.5 \mathrm{~g} / \mathrm{L}) \\
\text { 1-pentanol }(0.9 \mathrm{~g} / \mathrm{L})\end{array}$ & 5.1 & 40 & 2.1 & 8 & 0.2 & 0.22 & [87] \\
\hline $\begin{array}{c}\text { C. malaysiensis USMAA2-4 }{ }_{\mathrm{ABH} 16}{ }^{\mathrm{B}} \\
\text { Palm olein }(6.5 \mathrm{~g} / \mathrm{L}) \\
\text { 1-pentanol }(0.9 \mathrm{~g} / \mathrm{L})\end{array}$ & 5.4 & 69 & 3.7 & 7 & 0.3 & 0.33 & [87] \\
\hline $\begin{array}{l}\text { C. malaysiensis USMAA1020 } \\
\text { Oleic acid }(6.5 \mathrm{~g} / \mathrm{L}) \\
\text { 1-pentanol }(1.3 \mathrm{~g} / \mathrm{L})\end{array}$ & - & 76 & - & 10 & - & - & [88] \\
\hline
\end{tabular}


Table 2. Cont.

\begin{tabular}{|c|c|c|c|c|c|c|c|}
\hline \multirow{2}{*}{$\begin{array}{l}\text { Microorganisms and } \\
\text { Carbon Sources }\end{array}$} & \multirow{2}{*}{$\underset{(\mathrm{g} / \mathrm{L})}{\operatorname{Biomass}}$} & \multicolumn{2}{|c|}{ PHA Content } & \multicolumn{2}{|c|}{ 3HV Composition } & \multirow{2}{*}{$\begin{array}{l}\text { 3HV Yield } \\
\text { (g/g) }\end{array}$} & \multirow{2}{*}{ Ref. } \\
\hline & & $(w t \%)$ & $(g / L)$ & $(\mathrm{mol} \%)$ & $(g / L)$ & & \\
\hline $\begin{array}{l}\text { Erwinia sp. USMI-20 } \\
\text { Palm oil }(4.6 \mathrm{~g} / \mathrm{L}) \\
\text { 1-pentanol }(1.4 \mathrm{~g} / \mathrm{L})\end{array}$ & 4.8 & 62 & 3.0 & 20 & 0.6 & 0.43 & {$[60]$} \\
\hline $\begin{array}{l}\text { Massilia haematophila UMTKB-2 } \\
\text { Glucose }(16.0 \mathrm{~g} / \mathrm{L}) \\
\text { 1-pentanol }(1 \mathrm{~g} / \mathrm{L})\end{array}$ & - & - & 5.0 & 7 & 0.4 & 0.40 & [89] \\
\hline $\begin{array}{c}\text { Methylobacterium extorquens G10 } \\
\text { Methanol (fractional supply by } 5-20 \mathrm{~mL} \text { ) } \\
\text { 1-pentanol (fractional supply by } 2 \%-20 \% \\
\text { v/v methanol) }\end{array}$ & $25-40$ & $30-45$ & 7.5-18.0 & $14-50$ & $2.5-4.5$ & - & [90] \\
\hline $\begin{array}{l}\text { Methylocystis sp. WRRC1 } \\
\text { Methane gas }(75 \mathrm{~mL}) \\
\text { 1-pentanol }(1.0 \mathrm{~g} / \mathrm{L})\end{array}$ & - & - & 0.3 & - & 0.2 & 0.17 & [86] \\
\hline $\begin{array}{c}\text { Methyloligella halotolerans C2 } \\
\text { Methanol (5-20 mL fractional supply) } \\
\text { 1-pentanol (fractional supply by } 5-15 \% \mathrm{v} / \mathrm{v} \\
\text { methanol) }\end{array}$ & - & $49-98$ & - & $2-51$ & - & - & [91] \\
\hline $\begin{array}{c}\text { P. denitrificans ATCC } 17741 \\
\text { 1-pentanol (maintained at } 1.6 \mathrm{~g} / \mathrm{L})\end{array}$ & 6.8 & 18 & 1.2 & 100 & 1.2 & - & [14] \\
\hline \multicolumn{8}{|c|}{ Mixed precursors } \\
\hline $\begin{array}{c}\text { C. necator DSM } 545 \\
\text { Levulinic acid }(1.0 \mathrm{~g} / \mathrm{L}) \\
\text { Sodium propionate }(2.5 \mathrm{~g} / \mathrm{L})\end{array}$ & 1.0 & 33 & 0.3 & 73 & 0.2 & 0.24 & [65] \\
\hline $\begin{array}{c}\text { C. necator DSM } 545 \\
\text { Levulinic acid }(1.0 \mathrm{~g} / \mathrm{L}) \\
\text { Sodium propionate }(1.0 \mathrm{~g} / \mathrm{L})\end{array}$ & 0.5 & 19 & 0.1 & 78 & Trace & - & [10] \\
\hline $\begin{array}{c}\text { H. pseudoflava DSM } 1034 \\
\text { Whey permeate }(47.0 \mathrm{~mL} / \mathrm{L}) \\
\text { Levulinic acid } \\
(0.5 \mathrm{~g} / \mathrm{L}, \text { initial and } 3 \text { times feeding) } \\
\text { Sodium valerate } \\
(1.0 \mathrm{~g} / \mathrm{L} \text {, initial and } 3 \text { times feeding })\end{array}$ & 6.6 & 67 & 4.4 & 55 & 2.4 & 0.43 & [68] \\
\hline
\end{tabular}

Only the most promising condition was included for studies involving multiple cultivation conditions. Trace (concentration < $0.1 \mathrm{~g} / \mathrm{L}$ ). A C. glutamicum ATCC13869 transformant harboring C. necator phaCAB $B_{\text {Re }}$ genes. ${ }^{B}$ C. malaysiensis USMAA2-4 transformant harboring C. necator H16 lipAB genes. ${ }^{C}$ C. necator mutant with $\mathrm{P}(3 \mathrm{HB})-$ negative phenotype [92]. ${ }^{\mathrm{D}} \mathrm{H}$. seropedicae Z69 with the 2-methylcitrate synthase $(\operatorname{PrpC})$ gene eliminated.

\section{Techno-Economic and Sustainability Assessment}

The annual operating costs in PHA production generally include the direct fixed capital-dependent items, labor-dependent items, administration, and overhead expenses, raw materials, utilities, and downstream processing such as waste management. According to the techno-economic analysis conducted by Choi and Lee (1999) for various pure carbon sources, the substrate cost accounted for $48 \%-60 \%$ of the total costs (Figure 3) [93]. After excluding the trace elements, which are essentials, pure carbon sources that possess high nutritional value such as glucose, glycerol, starch, methane, oils, and volatile fatty acids are commercial products, and their employment leads to higher substrate cost compared to that of industrial or domestic wastes. Due to higher economic advantage and increasing emphasis on sustainability, the employment of wastes as carbon sources is widely attempted. Theoretically, substituting pure substrates with wastes contributes to a huge reduction in raw material expenses. However, pretreatments are needed for certain wastes to remove impurities and toxins or to adjust $\mathrm{pH}$ [94]. Pretreatments impose additional costs whereby extra chemicals or equipment are necessary with possible individual optimization. Bhattacharyya and co-workers (2015) reported decreased raw material cost to $39 \%$ with the employment of wheat stillage, but the utilities cost increased to $21 \%$ as compared to that reported by Choi and Lee (1999) (Figure 3) [93,95]. 


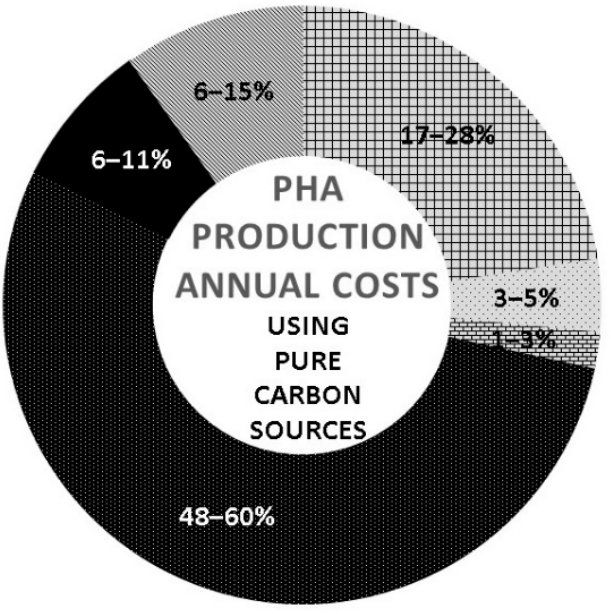

\# Direct-fixed capital-dependent cost 宦 Administration and overhead

-Utilities

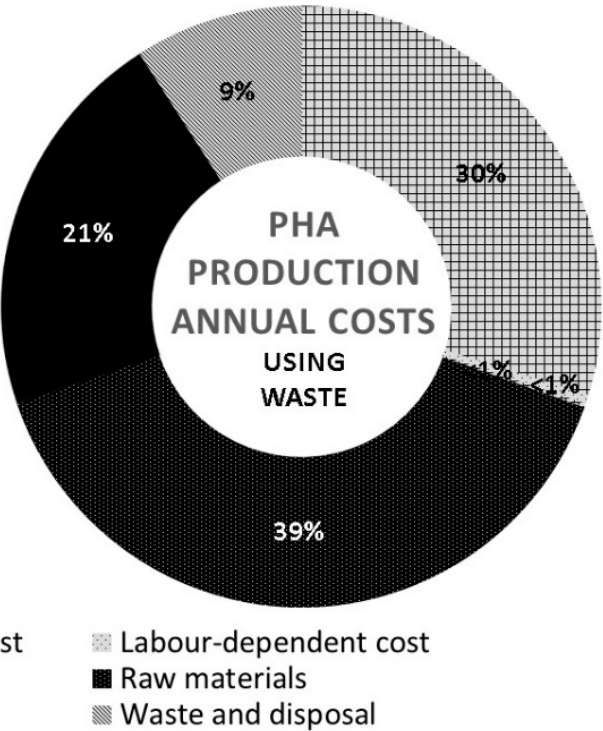

Figure 3. Techno-economic analysis on PHA production annual costs using pure carbon sources and wastes $[93,95]$.

As opposed to main carbon sources, where numerous studies have been conducted on various wastes, employing wastes as $3 \mathrm{HV}$ precursors is not practical due to the composition inconsistency [95]. Due to the necessity of propionyl-CoA for $3 \mathrm{HV}$ formation, sole reliance on wastes results in the narrow choice to those with propionate or valerate related components; thus, in most cases, a $3 \mathrm{HV}$ precursor is still required to achieve sufficient $3 \mathrm{HV}$ fraction for the copolymer to be practically useful $[64,95]$. This leads to increased raw material cost as propionic acid and valeric acid, which are widely preferred by PHA-producing bacteria, are high-cost precursors (Table 2). The potential of 1-propanol and 1-pentanol as alternatives for propionic acid and valeric acid is well-known but lack practicality due to its high toxicity to the majority of bacteria. Since 1996, several PHA-producing bacteria from different genera have been reported to use 1-propanol or/and 1-pentanol as 3HV precursors (Table 2). The emergence of these bacteria bypasses the bottleneck of precursor dominance by organic acids and enables further innovation in fermentation strategies to develop economically feasible and sustainable production processes. Furthermore, 1propanol and 1-pentanol are manufactured through well-established oxo synthesis and can be biosynthesized by bacteria from sustainable carbon sources such as glucose, glycerol, and organic wastes, which are abundant in nature.

\section{Oxo Synthesis of Alkyl Alcohols}

Oxo synthesis is an established process for the manufacture of alkyl alcohols at an industrial scale with simple operational requirements and low specificity in raw materials, including branched-chain, long-chain, and cyclic olefins [96,97]. It is thoroughly investigated for the production of a wide variety of industrial chemicals. The synthesis involves hydroformylation to convert olefins (also known as alkenes) into aldehydes to be further converted into alcohols through hydrogenation. Homogeneous catalysts are employed in hydroformylation, while heterogenous catalysts are employed in hydrogenation for reaction induction. Generally, these reactions are carried out in separate reactors where the resulting aldehydes from the primary reactor are transferred into the second reactor to be hydrogenated. Catalysts and carbon monoxide in the primary reactor are removed either by decobalting or been recycled back to the primary reactor to prevent entry into the second reactor as a precautious measure to extend the shelf life of hydrogenation catalysts. Recycling the catalysts contributes to high economic feasibility as high-cost catalysts such as rhodium-based catalysts can be reused for subsequent batches. However, an $8-55 \%$ 
decrease in catalyst yield after repeated recycling is expected [98]. The resulting alcohols are purified from the mixture via distillation (Figure 4) [97].

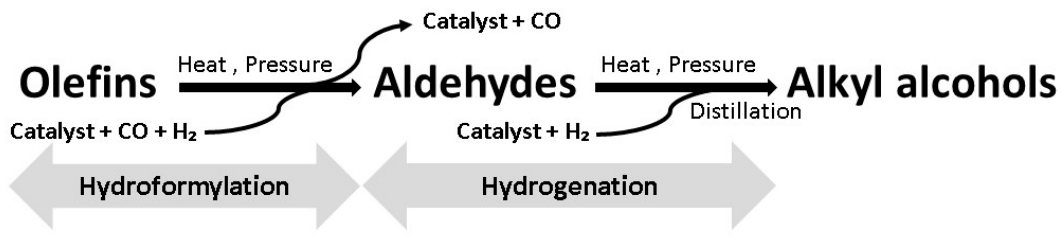

Synthesis of 1-propanol

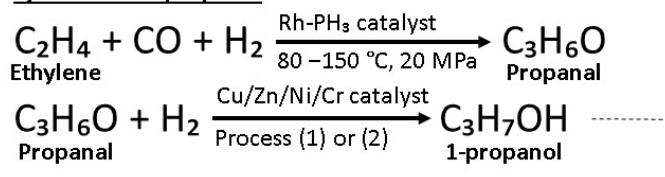

(1) Heterologous vapour phase:

110-150 ${ }^{\circ} \mathrm{C}$, 0.14-1.0 Mpa, $\mathrm{H}_{2}: \mathrm{C}_{3} \mathrm{H}_{6} \mathrm{O}(20: 1 \mathrm{~mol} / \mathrm{mol})$

(2) Heterologous liquid phase:

$95-120^{\circ} \mathrm{C}, 3.5 \mathrm{Mpa}$

Synthesis of 1-pentanol

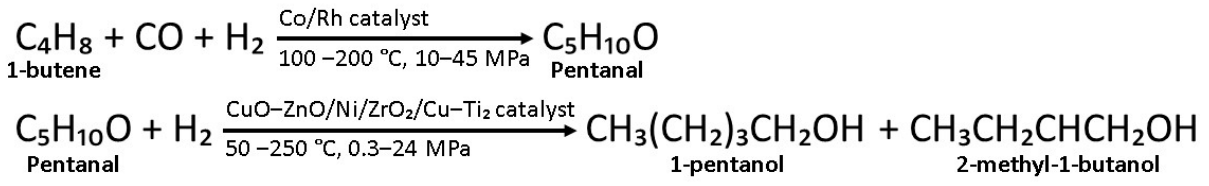

Figure 4. Oxo synthesis of alkyl alcohols $[62,97,99]$.

\subsection{1-Propanol}

Oxo synthesis of 1-propanol begins with the rhodium-catalyzed hydroformylation of ethylene (also known as ethene) to propanal with the aid of rhodium-triphenylphosphine catalysts. The resulting 1-propanal is distilled from the catalyst-containing solution, and carbon monoxide is removed. Hydrogenation can be carried out in either the heterogenous vapor phase or the heterogenous liquid phase. Heterogeneous vapor phase hydrogenation takes place at $110-150{ }^{\circ} \mathrm{C}$ and $0.14-1.00 \mathrm{MPa}$ with the aid of copper, zinc, nickel, and chromium catalysts supported on alumina (CAS:1344-28-1) or kieselguhr (CAS:91053-39-3) [62]. Heat is removed either by an external heat exchange device or an internal cooler [100]. This process produces impurities such as dipropyl ether, ethane, and propyl propionate. Selectivity enhancers such as alkali or transition metals are added to reduce the formation of esters, while an additional $1 \%-10 \%$ water could suppress the formation of ether [62,101]. Propyl propionate is separated from the product mixture and hydrogenolyzed with the aid of reduced $\mathrm{CuO}-\mathrm{ZnO}$ catalysts at $75-300{ }^{\circ} \mathrm{C}$ and $9.8 \mathrm{kPa}-9.8 \mathrm{MPa}$ to produce 1-propanol as the major product [62]. Heterologous liquid phase hydrogenation involved nickel or copper catalysts at a lower temperature of 95-120 ${ }^{\circ} \mathrm{C}$ and a higher pressure of 3.5 MPa. Crude 1-propanol is purified via distillation with the aid of an azeotroping agent such as dipropyl ether or cyclo-hexane to remove water for highly pure 1-propanol yield (>99\%) (Figure 4) [62,102].

\subsection{1-Pentanol}

Oxo synthesis of 1-pentanol begins with hydroformylation of 1-butene. Subsequent hydrogenation yields two $C_{5}$ products that are 1-pentanol and 2-methyl-1-butanol. For cobalt-catalyzed hydroformylation, the ratio of the product is 7:3 (1-pentanol:2-methyl-1butanol) after subsequent hydrogenation. When rhodium-triphenylphosphane is employed instead, a higher yield of 1-pentanol is achieved with a 9:1 (1-pentanol:2-methyl-1-butanol) ratio (Figure 4) [99].

\section{Biosynthesis of 1-Propanol and 1-Pentanol by Wild-Type Bacteria \\ 6.1. The Wood-Werkman Pathway in Propionibacteria}

Biosynthesis of 1-propanol by wild-type bacteria is inefficient as 1-propanol is synthesized as a byproduct through propionic acid synthesis processes. Propionibacteria such 
as Propionibacterium acidipropionici and Propionibacterium freudenreichii are able to produce 1-propanol through the Wood-Werkman pathway (also known as the dicarboxylic pathway, or the methylmalonyl-CoA pathway). The synthesis process requires an anaerobic condition where the carbon source is converted into pyruvate and enters the Wood-Werkman pathway to produce propionic acid as the main product $[103,104]$. The 1-propanol yield reported was in the range of $0.04-0.14 \mathrm{~mol} / \mathrm{mol}$, equivalent to $0.6-1.8 \mathrm{~g} / \mathrm{L}$. The 1-propanol production was found to be higher when glycerol was employed, compared to glucose $[105,106]$. The precise processes involved in 1-propanol formation are undetermined but could probably be by two-step reduction from propionyl-CoA to 1-propanol aided by acylating propionaldehyde dehydrogenase and propanol dehydrogenase (Figure 5) [107].

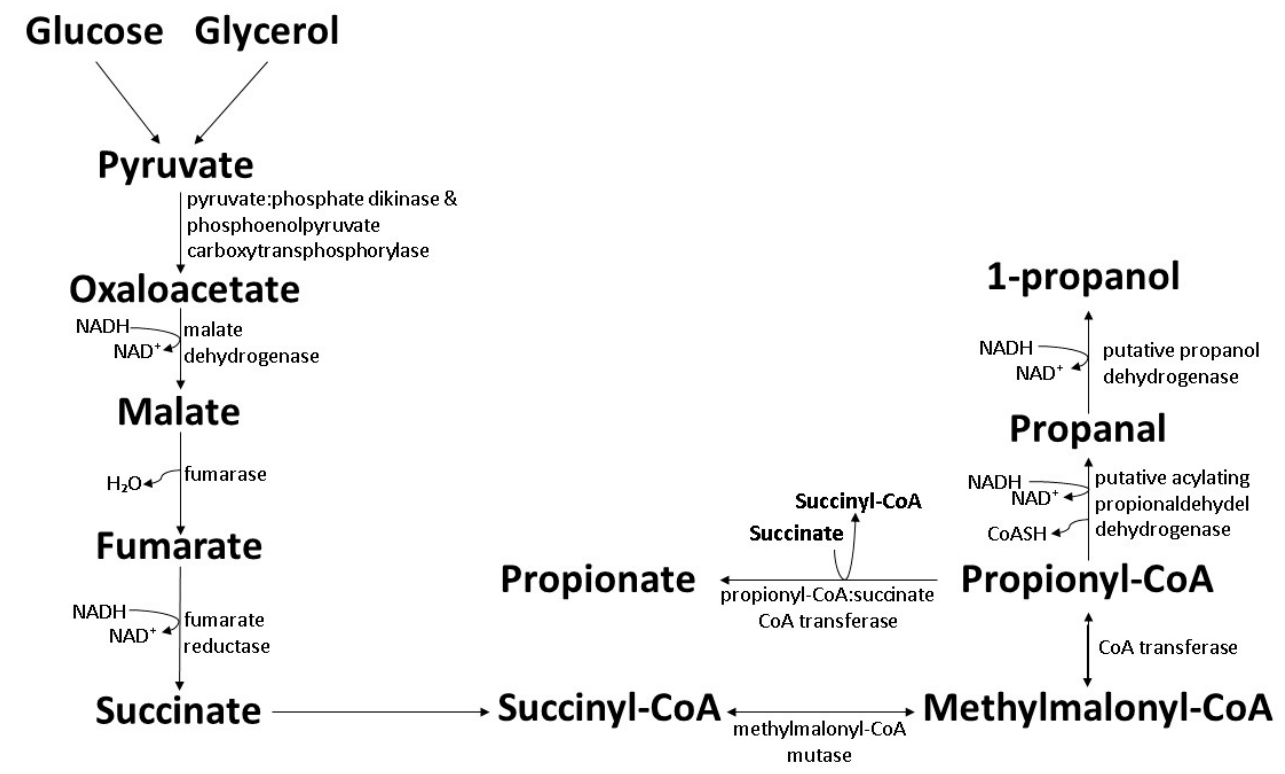

Figure 5. Biosynthesis of 1-propanol by wild-type Propionibacteria through the Wood-Werkman pathway $[103,104]$.

\subsection{The Acrylate Pathway in Clostridium}

Clostridium propionicumable and Clostridium neopropionicumable are able to use amino acids (alanine and serine), lactate, and ethanol as growth-promoting substances under anaerobic conditions $[108,109]$. C. neopropionicumable synthesizes a small amount of 1propanol $(0.06 \mathrm{~g} / \mathrm{L}, 0.03 \mathrm{~mol} / \mathrm{mol})$ from ethanol with propionate and acetate as the main products [109]. By employing the bacterial mixture dominated by Alkalibaculum bacchi (34\%) and C. propionicumable (54\%), C. propionicumable produced $6.0 \mathrm{~g} / \mathrm{L} 1$-propanol and $1.0 \mathrm{~g} / \mathrm{L}$ 1-butanol, whereas $A$. bacchi produced $8.0 \mathrm{~g} / \mathrm{L}$ ethanol from syngas (the carbon source) and corn-steep liquor (the source of amino acids and minerals) [110]. The resulting 1-propanol was proposed to be the product from a two-step reduction in propionyl-CoA produced through the acrylate pathway by using the lactoyl-CoA that is not used for propionic acid synthesis (Figure 6) [109]. However, further experimentations are needed to provide essential information for a complete view of the biosynthesis pathway. 


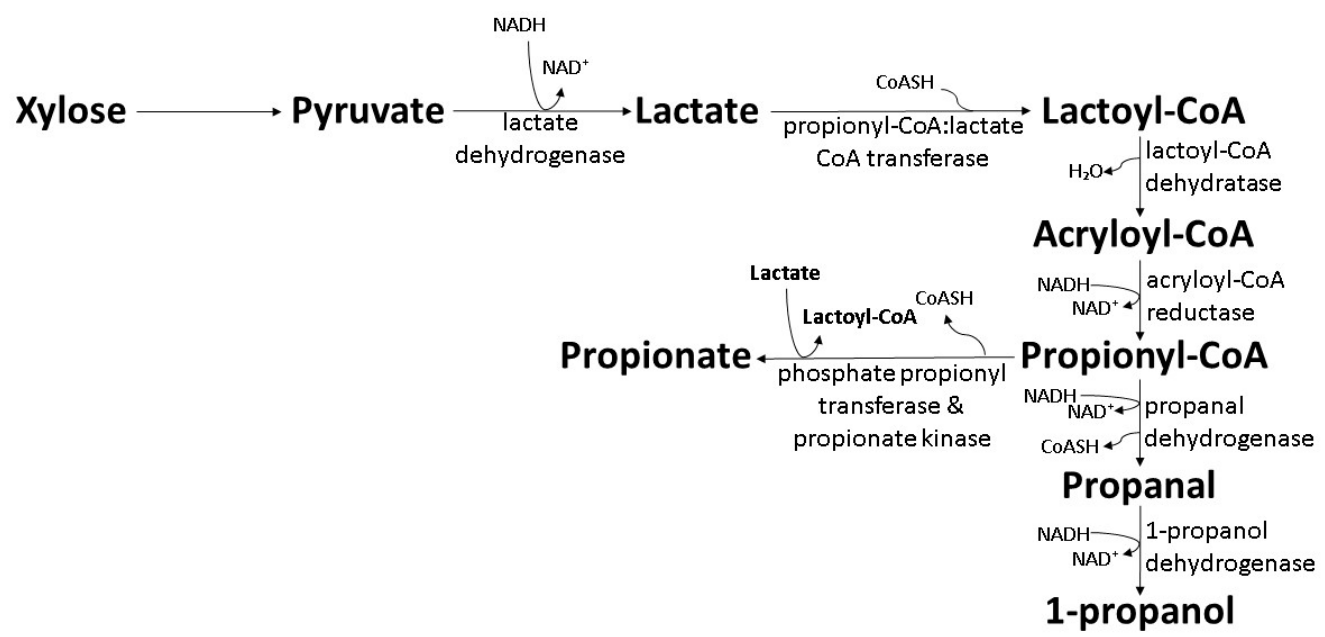

Figure 6. Biosynthesis of 1-propanol by wild-type C. propionicumable and C. neopropionicumable through the acrylate pathway $[109,110]$.

\subsection{The Carboxylate Reduction Pathway in Clostridium}

Anaerobic digestion by microbial consortia is a promising hydrogen production process where the members in the microbial community play different roles to convert raw materials into hydrogen under anaerobic conditions. As sterilization is commonly excluded from anaerobic digestion, organic acids produced by acetogens in the consortia cause decreased $\mathrm{pH}$ that disrupts the metabolic activity of hydrogen-producing bacteria [111]. Clostridium ragsdalei (ATCC BAA-622, DSM 15248) is an acetogen capable of synthesizing alcohols by ferredoxin-mediated carboxylate reduction. With the involvement of exogenous $\mathrm{CO}$ and ferredoxin, $n$-fatty acids up to six carbons in length can be reduced to corresponding alcohols (Figure 7). The concentration of produced 1-propanol reported was $1.7 \mathrm{~g} / \mathrm{L}$ 1-propanol from propionic acid, with a conversion efficiency of $97 \%$. However, the concentration of 1-pentanol obtained was merely $0.2 \mathrm{~g} / \mathrm{L}$, with a conversion efficiency of $82 \%$ [112].

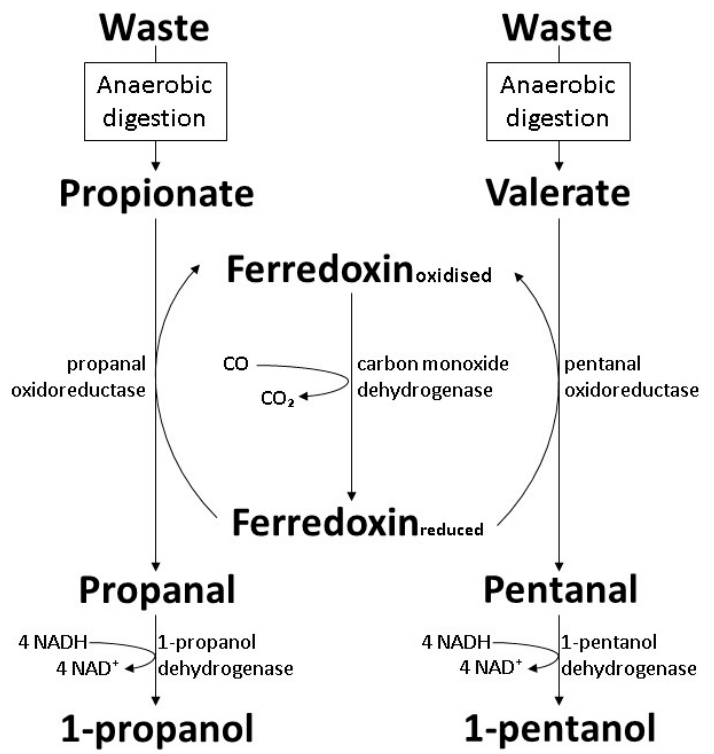

Figure 7. Biosynthesis of 1-propanol and 1-pentanol by wild-type C. ragsdalei through the carboxylate reduction pathway [112]. 


\section{Biosynthesis of 1-Propanol and 1-Pentanol by Genetic-Engineered E. coli}

\subsection{Co-Expression of the Citramalate and Threonine Pathway}

Numerous genetic engineering attempts were carried out for alkyl alcohols biosynthesis through the individual threonine or citramalate pathway and showed successful biosynthesis of 1-propanol from the intermediate 2-ketobutyrate in the pathways [113,114]. For greater industrial applicability, co-expression of both pathways was attempted in E. coli BW25113. The simultaneous operation of the pathways in a single host showed a synergic effect on 1-propanol production. The co-expression provided a larger 2-ketobutyrate pool for decarboxylation and reduction to 1-propanol (Figure 8). A high 1-propanol concentration of $8.0 \mathrm{~g} / \mathrm{L}$ was reported with a 1-propanol yield of $0.15 \mathrm{~g} / \mathrm{g}$ from glucose, which was higher than 0.09 and $0.11 \mathrm{~g} / \mathrm{g}$ for individual threonine and citramalate pathway, respectively [115].

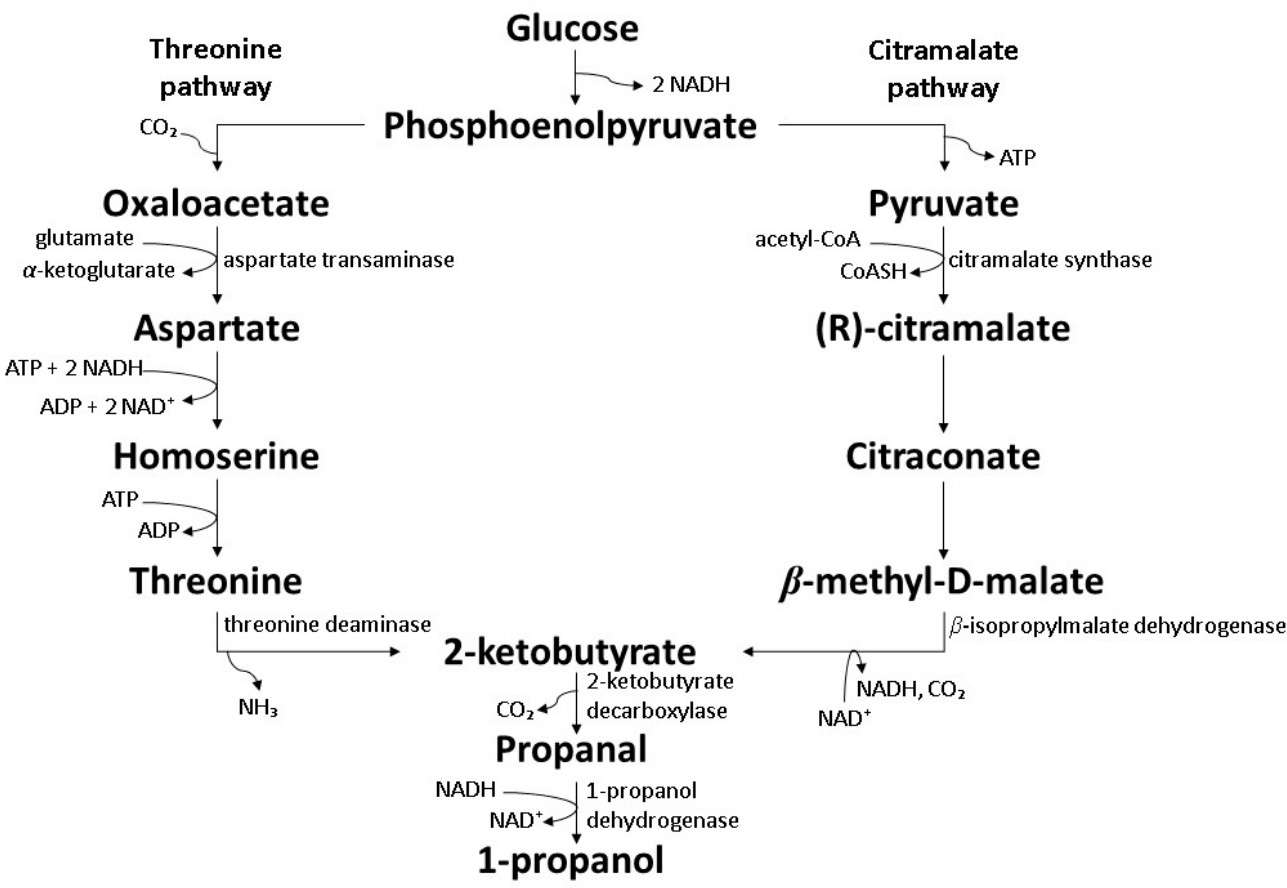

Figure 8. Biosynthesis of 1-propanol by genetically engineered E. coli BW25113 through co-expression of the citramalate and threonine pathway [115].

\subsection{Interactive Elongation Cycle of 2-Ketoacids}

Biosynthesis of 1-pentanol was made possible by introducing Lactococcus lactis ketoisovalerate decarboxylase (Kivd) modified via saturated mutagenesis of the V461 key residue of the enzyme with glycine and serine into E. coli BW25113 to promote its selectivity toward 2-ketocaproate, which is the precursor for 1-pentanol. Besides lowered catalytic efficiency of the modified Kivd toward 2-ketoacids upstream of 2-ketocaproante, the increased supply of acetyl-CoA by acetate feeding encouraged 2-ketoacid elongation cycle for enhanced 1-pentanol production (Figure 9). The high specificity of this approach was implied by $90 \%$ 1-pentanol in the alcohol product mixture, equivalent to $2.2-2.4 \mathrm{~g} / \mathrm{L}$ upon production harvest. The synthesis of alcohols with a longer alkyl chain was found to be minimized as further elongation of the 2-ketoacid was discouraged due to the active use of 2-kerocaproate for 1-pentanol synthesis [116]. 


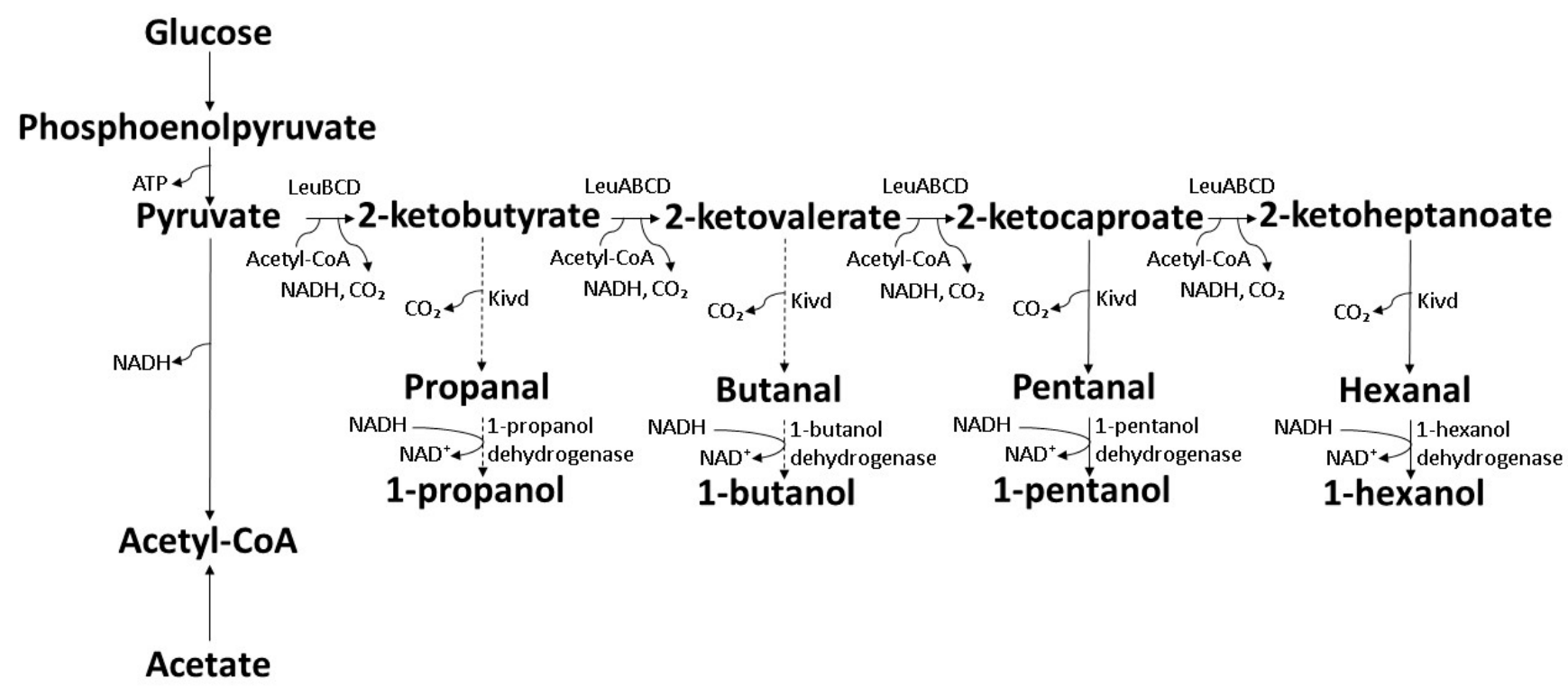

Figure 9. Biosynthesis of 1-pentanol by genetically engineered E. coli BW25113 through interactive elongation cycles of 2-letoacids [116]. Dash arrow indicates lower selectivity toward the reaction.

\subsection{Extended Dissimilation of Succinate}

The sleeping beauty mutase (SBM) operon in E. coli is a four-gene operon (sbm-ygfD$y g f G-y g f H$ ) that encodes various enzymes required in a cobalamin-dependent metabolic pathway for decarboxylation of succinate into propionate [117]. An activated chromosomal SBM operon encodes methylmalonyl-mutase (by sbm), methylmalonyl-CoA decarboxylase (by $y g f G$ ), and propionyl-CoA:succinate CoA transferase (by $y g f H$ ) in plasmid-free propanogenic E. coli BW25113 enabled extended dissimilation of succinate to synthesis 1-propanol (Figure 10). Glycerol favored solventogenesis over glucose due to the necessity of a solventogenic pathway as an auxiliary channel for redox balance upon glycerol dissimilation under anaerobic conditions. An anaerobic fed-batch strategy established by using the engineered E. coli strain produced high titers of $7.0 \mathrm{~g} / \mathrm{L}$ 1-propanol, thus implying its high industrial applicability [118].

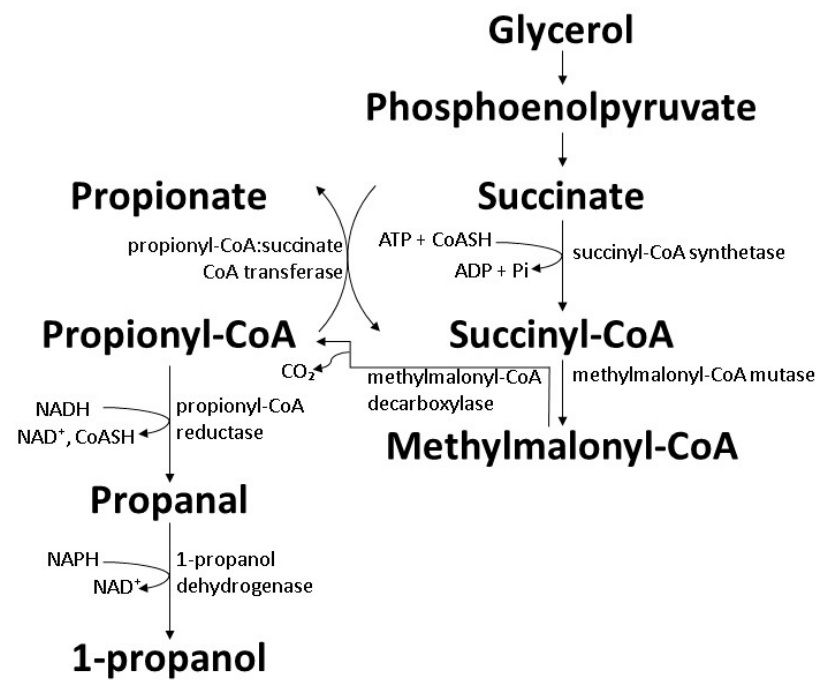

Figure 10. Biosynthesis of 1-propanol by genetically engineered E. coli BW25113 with activated SBM operon for extended dissimilation of succinate [118]. 


\subsection{Acquired Carboxylate Reduction Pathway}

Conversion of organic acids produced by acetogens during anaerobic digestion into other useful products is suggested to be beneficial as a solution to maintain the stability of the biogas production process. An E. coli BL21(DE3) strain harboring Clostridium acetobutylicum alcohol dehydrogenase (AdhE2) and Megasphaera hexanoica acyl-CoA transferase (ACT01_02765) was developed for conversion of the $C_{2}-C_{8}$ organic acids commonly found in anaerobic digestion into corresponding primary alcohols. The metabolic pathway is relatively simpler as it only involves two steps aided by two enzymes (Figure 11). Following the conversion rate of 1.1 for $\mathrm{C}_{4}$ acid into 1-butanol, the functional alcohol dehydrogenase and acyl-CoA transferase resulted in a promising conversion rate of 0.8 for both 1-propanol and 1-pentanol [119].

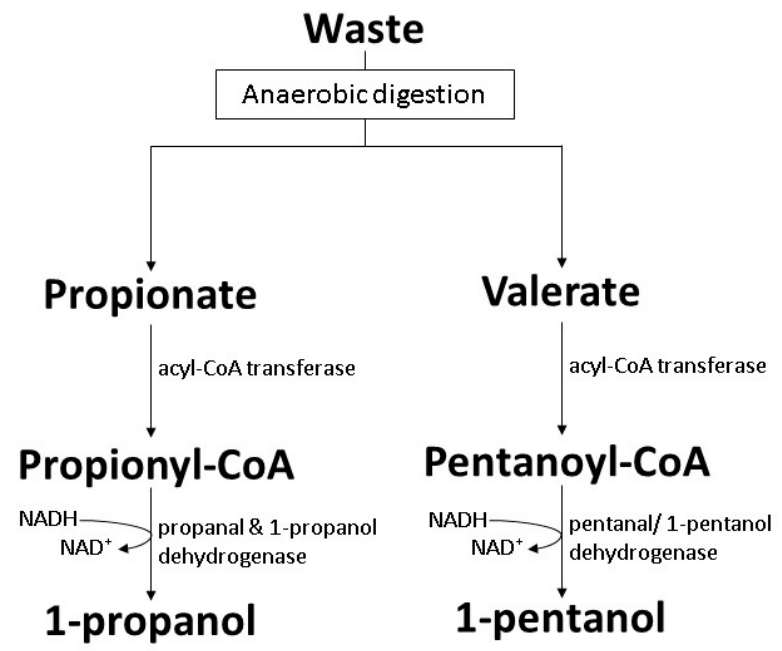

Figure 11. Biosynthesis of 1-pentanol by genetically engineered E. coli BL21(DE3 with acquired carboxylate reduction pathway [119].

\section{Alkyl Alcohol-Tolerant $\mathrm{P}(3 \mathrm{HB}-\mathrm{co}-3 \mathrm{HV})$-Producing Bacteria}

Alcohols are unsuitable to be employed as $3 \mathrm{HV}$ precursors for $C$. necator (also known as Ralstonia eutropha, Alcaligenes eutrophus, or Wautersia eutropha), which is the standard PHAproducing bacteria. Although C. necator $\mathrm{H} 16$ is capable of surviving methanol, ethanol, and propanol, extensive exposure to these alcohols is detrimental to PHA accumulation, thus resulting in lower biomass. The employment of $8.0 \mathrm{~g} / \mathrm{L}$ 1-propanol, which is convertible into propionyl-CoA, contributed to merely $3 \mathrm{~mol} \% 3 \mathrm{HV}$ with a $3 \mathrm{HV}$ yield of $0.14 \mathrm{~g} / \mathrm{g}[13,120]$ (Table 2). The individual employment of 1-propanol and 1-pentanol also caused a remarkably high reduction in biomass and PHA content of $C$. necator DSM 545. The employment of 1-pentanol caused C. necator DSM 545 biomass and PHA content to decrease by $40 \%$ and $20 \%$, respectively. Comparatively,1-propanol exerted a lower adverse effect compared to 1-pentanol, whereby its employment decreased C. necator DSM 545 PHA content by $10 \%$ with no negative influence on bacterial biomass [10] (Table 2). To overcome the limitation in $3 \mathrm{HV}$ precursor selection, isolation of alkyl alcohol-tolerant $\mathrm{P}(3 \mathrm{HB}-\mathrm{co}-3 \mathrm{HV})$-producing bacteria is continuously attempted and has led to the discovery of various promising bacteria with the capability to use alkyl alcohols as $3 \mathrm{HV}$ precursors (Figure 12). 


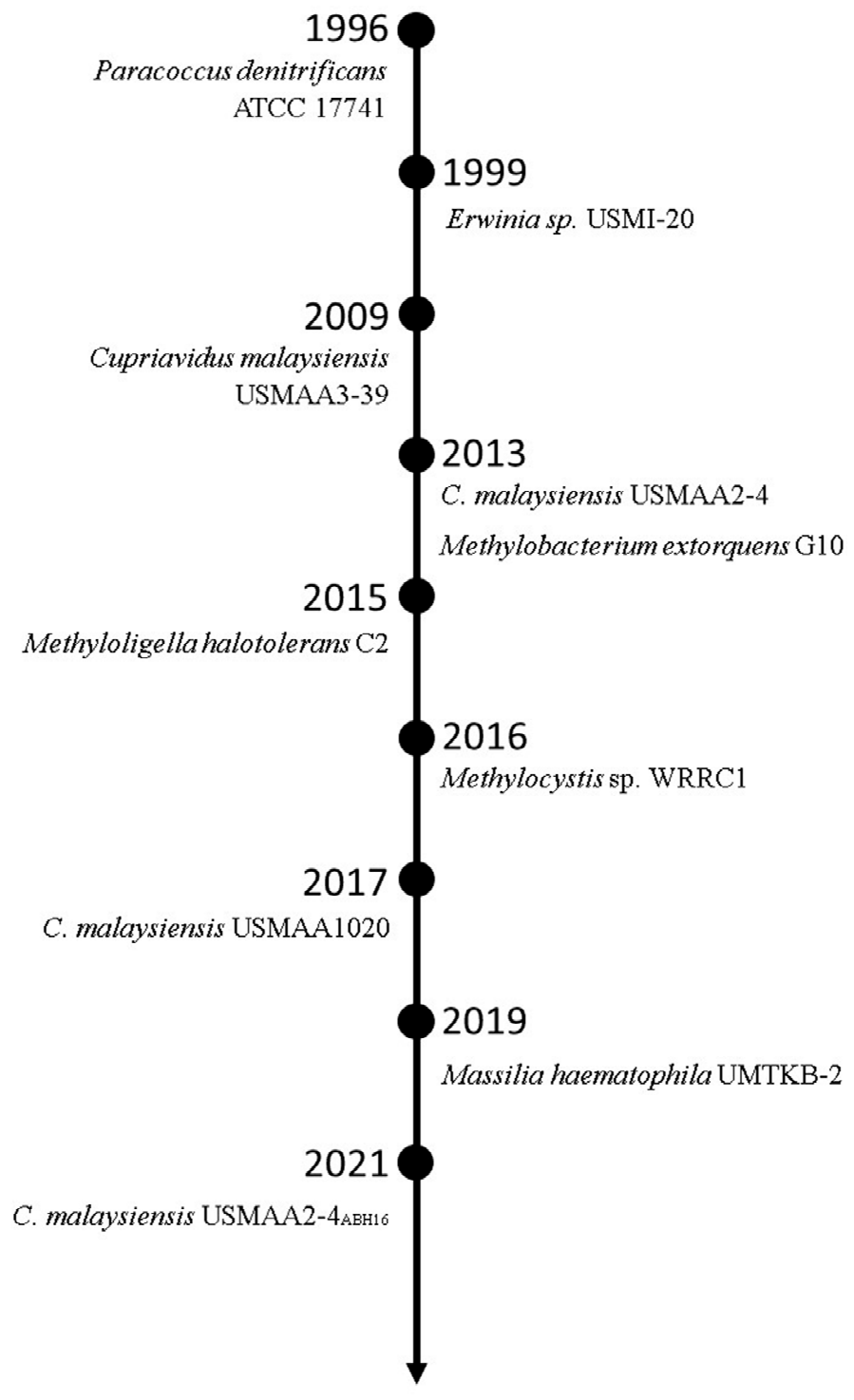

Figure 12. Timeline of the emergence of alkyl alcohol-tolerant $\mathrm{P}(3 \mathrm{HB}-\mathrm{co}-3 \mathrm{HV})$-producing bacteria.

P. denitrificans ATCC 17741 was the first bacteria reported in 1996 for the use of alkyl alcohol as the $3 \mathrm{HV}$ precursor. P. denitrificans ATCC 17741 is a mixotrophic colorless sulfur bacterium capable of using 1-pentanol as the sole carbon source for growth and $\mathrm{P}(3 \mathrm{HV})$ accumulation $[14,121]$. The study was conducted by maintaining the concentration of 1-pentanol at $1.6 \mathrm{~g} / \mathrm{L}$ for $24 \mathrm{~h}$. Approximately $6.8 \mathrm{~g} / \mathrm{L}$ biomass with $1.2 \mathrm{~g} / \mathrm{L} \mathrm{P}(3 \mathrm{HV})$ homopolymer was achieved [14] (Table 2).

Erwinia sp. USMI-20 was reported with its preference for alkyl alcohols instead of organic acids as 3HV precursors. Erwinia sp. USMI-20 achieved higher biomass with the co-employment of 1-propanol and 1-pentanol compared to that when palm oil was employed solely. A higher PHA content of 50 and $62 \mathrm{wt} \%$ was also achieved for 1-propanol and 1-pentanol, respectively, compared to $40 \mathrm{wt} \%$ and $34 \mathrm{wt} \%$ for propionic acid and valeric acid. 1-pentanol was more promising compared to 1-propanol as Erwinia sp. USMI-20 accumulated a higher $3 \mathrm{HV}$ fraction of $20 \mathrm{~mol} \%$ from 1-pentanol compared to $6 \mathrm{~mol} \%$ from 1-propanol. 1-pentanol can be employed as a substitute for valeric for Erwinia sp. USMI-20 owing to the higher $3 \mathrm{HV}$ yield of $0.43 \mathrm{~g} / \mathrm{g}$ for 1-pentanol, which was 2-fold higher than that for valeric acid [60]. The production was scaled up to $10 \mathrm{~L}$ by employing $4.6 \mathrm{~g} / \mathrm{L}$ palm oil and $1.4 \mathrm{~g} / \mathrm{L}$ 1-pentanol, where 1-pentanol was added at $20 \mathrm{~h}$ post incubation. The $3 \mathrm{HV}$ 
fraction achieved was $20 \mathrm{~mol} \%$ in $56 \mathrm{wt} \%$ PHA content of $5.4 \mathrm{~g} / \mathrm{L}$ biomass, with $0.43 \mathrm{~g} / \mathrm{g}$ $3 \mathrm{HV}$ yield [122] (Table 2 ).

Despite the negative influence observed for C. necator, there are several Cupriavidus sp. that are capable of using alkyl alcohols as $3 \mathrm{HV}$ precursors with no adverse effect on either bacterial biomass or PHA accumulation. C. malaysiensis USMAA2-4, C. malaysiensis USMAA1020, and C. malaysiensis USMAA9-39 are three PHA-producing bacteria favoring alkyl alcohols over organic acids for 3HV formation [123]. C. malaysiensis USMAA2-4 and C. malaysiensis USMAA1020 were able to accumulate $7-10$ mol\% $3 \mathrm{HV}$ from 1-pentanol [87,88,123-125] (Table 1). The 3HV yield of C. malaysiensis USMAA2-4 and its transformant strain harboring C. necator H16 lipAB genes was $0.22 \mathrm{~g} / \mathrm{g}$ and $0.33 \mathrm{~g} / \mathrm{g}$, respectively, which were both higher than $0.14 \mathrm{~g} / \mathrm{g}$ for C. necator $\mathrm{H} 16[13,87,88]$. The sole employment of 1-pentanol resulted in a higher C. malaysiensis USMAA9-39 PHA content of $46 \mathrm{wt} \%$ compared to $37 \mathrm{wt} \%$ for valeric acid. Despite the 1 -fold lower $C$. malaysiensis USMAA9-39 biomass resulting from the co-employment of 1-pentanol with oleic acid, the $3 \mathrm{HV}$ yield of $0.44 \mathrm{~g} / \mathrm{g}$ from 1-pentanol was comparable to $0.42 \mathrm{~g} / \mathrm{g}$ from valeric acid and a high 3HV composition of $24 \mathrm{~mol} \%$ was achieved [76] (Table 2).

M. extorquens $\mathrm{G} 10$ demonstrated the production of $\mathrm{P}(3 \mathrm{HB}-\mathrm{co}-3 \mathrm{HV})$ from an alkyl alcohol mixture of $\mathrm{C} 1$ and $\mathrm{C} 5$ alcohol. A $4 \mathrm{~L}$ production of $\mathrm{P}(3 \mathrm{HB}-\mathrm{co}-3 \mathrm{HV})$ from a methanolpentanol mixture by $M$. extorquens G10 showed a promisingly high PHA concentration of 7.5-18.0 g/L. The carbon mixture was supplemented fractionally based on the dissolved oxygen peaks observed. With an increased portion of 1-pentanol from 2 to $20 \mathrm{~mol} \%$, the biomass decreased with association to reduction in PHA content from 40.0 to $25.0 \mathrm{~g} / \mathrm{L}$ and 45 to $30 \mathrm{wt} \%$, respectively. Despite the negative influence on biomass and PHA content, $3 \mathrm{HV}$ composition of $14-50 \mathrm{~mol} \%$ was achieved [90] (Table 2).

M. halotolerans $\mathrm{C} 2$ demonstrated $\mathrm{P}(3 \mathrm{HB}-\mathrm{co}-3 \mathrm{HV})$ production from $\mathrm{C} 1, \mathrm{C} 2$, and $\mathrm{C} 5$ alkyl alcohol. $\mathrm{P}(3 \mathrm{HB}-c o-3 \mathrm{HV})$ production by $\mathrm{M}$. halotolerans $\mathrm{C} 2$ through fractional feeding of methanol-ethanol mixture resulted in increased 3HV composition from 2 to $51 \mathrm{~mol} \%$ parallel to increased 1-pentanol supply from 5 to $15 \% \mathrm{v} / \mathrm{v}$ methanol. A considerably high PHA content of 73-98 $\mathrm{wt} \%$ was accumulated by the bacterium [91] (Table 2).

$\mathrm{P}(3 \mathrm{HB}-\mathrm{co}-3 \mathrm{HV})$ production by Methylocystis sp. WRRC1 from methane and 1-pentanol demonstrated a $0.17 \mathrm{~g} / \mathrm{g} 3 \mathrm{HV}$ yield from $1.0 \mathrm{~g} / \mathrm{L} 1$-pentanol. The 6 -fold lower consumption of methane by the bacteria with the co-employment of 1-pentanol compared to that of sole employment of methane denoted the preference of the bacteria for 1-pentanol over methane. However, 1-pentanol is non-competitive against valerate where Methylocystis sp. WRRC1 achieved a 1-fold higher $3 \mathrm{HV}$ concentration with the co-employment of sodium valerate compared to that of 1-pentanol. On the other hand, the co-employment of sodium valerate did not cause reduced methane consumption and contributed to a higher $3 \mathrm{HB}$ concentration [86] (Table 2).

M. haematophila (also known as Naxibacter haematophila) UMTKB-2, a slow-growing bacterium, was also reported with the capability to use 1-pentanol for $3 \mathrm{HV}$ accumulation with a preference for 1-pentanol over valeric acid and sodium valerate. The co-employment of 1-pentanol resulted in 2-fold and 11-fold higher biomass and PHA content compared to that of valeric acid and sodium valerate, respectively. Upon optimization by using response surface methodology, M. haematophila UMTKB-2 achieved $7 \mathrm{~mol} \% 3 \mathrm{HV}$ with $0.40 \mathrm{~g} / \mathrm{g} 3 \mathrm{HV}$ yield. Unlike the PHA accumulation process of Cupriavidus sp. that ends within $48-72 \mathrm{~h}$, $122 \mathrm{~h}$ was needed for optimum $\mathrm{P}(3 \mathrm{HB}-\mathrm{co}-3 \mathrm{HV})$ accumulation by $M$. haematophila UMTKB2 [89] (Table 2).

\section{Mode of Action of 1-Propanol and 1-Pentanol on Proteins}

Short-chain alcohols exert a hydrophobic effect by interacting with proteins and lead to the structural unfolding of the protein [126]. Changes in membrane fluidity ensue due to the direct insertion of lipophilic agents into the cellular membrane after direct physicochemical interaction with alcohols. This induces adaptive membrane alteration by changing the fatty acid composition of the membrane [127]. Impaired inner membrane integrity associated 
with depletion in proton motive force due to the increased proton motive force demand for chemical, osmotic and mechanical adjustment induces the $p s p$ operon to prevent proton loss. As a result, the cells experience a metabolic shift to anaerobic respiration together with downregulation of motility for adjustment and maintenance of energy as well as for proton motive force usage [128]. The extent of water exclusion is greater with increasing alkyl groups of the alcohol, which is non-polar. By considering the hydrophobic effect of methanol $<$ ethanol $<$ propanol $=$ butanol, pentanol may exert a similar hydrophobic effect on protein and result in pentanol-induced protein unfolding [126]. Furthermore, pentanol is capable of inactivating membrane proteins such as transporters but rarely causes structural changes to the cell membrane [129].

\section{Mechanisms Involved in Alcohols Tolerance}

Aliphatic alcohols, aromatic compounds, or other organic solvents are toxic to bacteria when present in high concentrations. Nevertheless, certain bacteria are able to thrive in the high concentration of such toxic organic chemicals. Bacterial solvent tolerance is a multifactorial process that involves gene expression and subsequent physiological changes to respond to stress conditions. Extrusion of the toxic compounds from the cell to the external environment and reduced cell membrane permeability to prevent further influx of toxic compounds are the relevant mechanisms to survive alcohol stress.

\subsection{Changes in the Cell Membrane}

Alcohol-induced cell leakage of magnesium and nucleotides is the primary damaging action that affects bacterial viability in alcohols [130]. As alcohols interact with the cell membrane and decrease the degree of membrane organization, proteins that participate in membrane structure organization and surface stabilization are critical in alcohol tolerance [131]. Isomerase incorporates fatty acids into the phospholipid headgroups of the phospholipid bilayer and causes isomerization of cis unsaturated fatty acids to trans unsaturated fatty acids to form a denser membrane, as demonstrated by Pseudomonas and Vibiro [132]. Changes in cell membrane composition that attributed to increased cis-11 vaccenic acid (18:1) or cis-9 oleic acid (18:1) with a corresponding decrease in palmitic acid (16:0) were demonstrated for E. coli, Lactobacillus homohiochii, and Saccharomyces cerevisiae $[131,133,134]$. The synthesis of phosphatidylethanolamine by Zymomonas mobilis was partially inhibited in the presence of alcohols. As a result, a membrane with an elevated proportion of acidic phospholipids (phosphatidylglycerol and cardiolipin) and an overall reduction in the phospholipid:protein ratio is synthesized, thus increasing the efficiency of efflux pumps in alcohol extrusion $[135,136]$.

\subsection{Stress Response System}

Exposure to alkyl alcohols leads to changes in the level of expression of certain genes as responses to stress for adaptation. As demonstrated in E. coli, exposure to 1-butanol causes downregulation of several genes related to histidine, leucine, arginine, tryptophan, and methionine biosynthesis and transport, thus leading to a significantly lower level of related proteins. Downregulation of genes related to amino acids metabolism is an indicator for bacterial growth inhibition in alcohols. As opposed to that, opp operon (oppABCDF) that encodes the components in a polyamine-induced oligopeptide $A B C$ transport system is upregulated for the transport of hydrophilic substances to compensate for the hydrophobic pressure exerted by alcohols $[137,138]$. Genes responsible for response to heat shock and extracytoplasmic stress (cpx regulon) are upregulated, and periplasmic chaperone Spy is encoded to respond to protein misfolding activity [139-142]. Increased isobutanol tolerance of $C$. acetobutylicum is also conferred to overexpression of genes related to heat shock $[139,143]$. Genes related to the membrane and periplasmic space carbohydrate transport and metabolisms are upregulated to transport and phosphorylate hexoses and release the phosphate esters into the cytoplasm, probably as a repair mechanism for damaged bilayer $[139,144]$. Furthermore, the upregulation of genes from the 13-member nuo operon 
and 5-member cyo operon is also an indicator for the increased requirement of energy or disruption of respiratory efficiency upon exposure to 1-butanol [139]. However, the operons are downregulated when exposed to isobutanol [137]. Exposure to ethanol causes induced expression of $p s p$ operon to restore proton motive force lost due to disruption of the cell membrane by ethanol, but the expression level remains unchanged for isobutanol $[128,137]$.

\section{Challenges in Wide Implementation of Alkyl Alcohols as 3HV Precursors}

Low alcohol tolerance due to alcohol toxicity is the major drawback for the employment of alkyl alcohols as $3 \mathrm{HV}$ precursors. Isolation of novel PHA-producing bacteria with substantial alcohol tolerance is a continuous effort in developing production processes with higher economic feasibility. With established primary alkyl alcohol bioproduction processes, the employment of alkyl alcohols also contributes to sustainability. Alcohol tolerance involves complex regulatory systems, and knowledge from cell-wide stress response is still in demand. Theoretically, genetic engineering can be adopted to create an alkyl alcohol-tolerant PHA-producing bacteria by either introducing pha genes into an alkyl alcohol-tolerant host or modulating alcohol tolerance of a non-alkyl alcohol-tolerant PHA-producing bacteria. Comparatively, the former approach is more rational as alcohol tolerance involves complex systems and is not economically feasible for commercial importance.

Although genetic-engineered E. coli with mutated rpoA gene was constructed successfully to produce products with commercial importance such as 1-butanol, the attempt was based on extensive studies on the rpoA gene and its roles in phenotypic changes of E. coli [145-149]. Owing to numerous studies on the incorporation of pha genes into $E$. coli, which demonstrated successful production of various PHA, such approaches can be adopted for the construction of alkyl alcohol-tolerant strains with acquired PHA-producing ability [150-152]. However, a candidate strain with broad substrate preference is preferred for production process establishment with different substrates and fermentation strategies. The capability to use wastes with high carbon content will be an added value for higher industrial applicability owing to its sustainability and higher economic feasibility compared to pure carbon sources [87].

Despite the promising potential shown by the known alkyl alcohol-tolerant $\mathrm{P}(3 \mathrm{HB}-$ co-3HV)-producing bacteria, scaling up the production remains challenging. As low $3 \mathrm{HV}$ compositions are commonly reported for shake flask scale production, various production strategies have to be adopted to increase the molar fraction of $3 \mathrm{HV}$. Fed-batch production strategies that enable the addition of alkyl alcohols eventually are practically preferred to achieve high $3 \mathrm{HV}$ composition of $\mathrm{P}(3 \mathrm{HB}-\mathrm{co}-3 \mathrm{HV})$ and at the same time minimize the negative influences caused by the relative toxicity of alkyl alcohol. However, some of the bacteria that depicted decreased biomass and PHA content with the employment of alkyl alcohol at low concentration or with a preference for organic acid sodium salt over alkyl alcohol have low applicability as candidate $\mathrm{P}(3 \mathrm{HB}-\mathrm{co}-3 \mathrm{HV})$ producers when alkyl alcohols are to be employed. In addition, more studies on large-scale $\mathrm{P}(3 \mathrm{HB}-\mathrm{co}-$ $3 \mathrm{HV}$ ) production involving alkyl alcohols are still in demand to compare their industrial practicality as alternative $3 \mathrm{HV}$ precursors for organic acids in terms of sustainability and economic feasibility.

\section{Concluding Remark}

The high sale price of $\mathrm{P}(3 \mathrm{HB}-\mathrm{co}-3 \mathrm{HV})$ has been the major obstacle to commercialization. Although various carbon sources have been explored, limited precursor choice due to the domination by propionate and valerate has caused the development of diverse $\mathrm{P}\left(3 \mathrm{HB}-\mathrm{co}^{-}\right.$ $3 \mathrm{HV}$ ) production to reach a bottleneck. With increasing studies reporting the discovery of alkyl alcohol-utilizing PHA-producing bacteria with promising bioconversion efficiency of 1-propanol and 1-pentanol into 3HV, the toxicity of alkyl alcohols and low $3 \mathrm{HV}$ yield are no longer the major concern. Future attempts should focus on continuous searching of alkyl alcohols tolerant PHA-producing bacteria to discover more promising wild-type strains. 
Moreover, genetic engineering of bacterial metabolic pathways to achieve successful or higher bioconversion rate of alkyl alcohols into $3 \mathrm{HV}$ is also important to overcome low bacterial viability and alcohol-3HV bioconversion efficiency. However, more studies are required for techno-economic assessment to compare to what extent 1-propanol and 1pentanol could contribute to higher economic feasibility than propionate and valerate.

Author Contributions: Conceptualization, investigation, data curation, formal analysis, visualization, writing —original draft, review and editing, H.S.J.W.; writing—review and editing, K.B.; supervision, writing - review and editing, A.-A.A.A. All authors have read and agreed to the published version of the manuscript.

Funding: This research was funded by the Fundamental Research Grant Scheme (FRGS) (ref: FRGS/1/2021/STG01/USM/01/2) from the Ministry of Higher Education, Malaysia.

Institutional Review Board Statement: Not applicable.

Informed Consent Statement: Not applicable.

Conflicts of Interest: The authors declare no conflict of interest to disclose.

$\begin{array}{ll}\text { Abbreviations } & \\ \text { 3HB } & \text { 3-hydroxybutyrate } \\ \text { 3HV } & \text { 3-hydroxyvalerate } \\ \alpha-\mathrm{P}(3 \mathrm{HB}) & \text { Synthetic atactic poly(3-hydroxybutyrate) } \\ \text { Ag/BSA } & \text { Bovine serum albumin capped silver } \\ \text { AS } & \text { Ascorbic acid } \\ \text { CNC } & \text { Cellulose nanocrystals } \\ \text { CNT } & \text { Carbon nanotubes } \\ \text { DCP } & \text { Dicumyl peroxide } \\ \text { HA } & \text { Hydroxyapatite } \\ \text { HDPE } & \text { High-density polyethylene } \\ \text { LDPE } & \text { Low-density polyethylene } \\ \text { LLDPE } & \text { Linear low-density polyethylene } \\ \text { MAT } & \text { Organophilic attapulgite } \\ \text { mPEG } & \text { Monomethoxy poly(ethylene glycol) } \\ \text { NA } & \text { Not available } \\ \text { P(3HB) } & \text { Poly(3-hydroxybutyrate) } \\ \text { P(3HV) } & \text { Poly(3-hydroxyvalerate) } \\ \text { P(3HB-co-3HV) } & \text { Poly(3-hydroxybutyrate-co-3-hydroxyvalerate) } \\ \text { PBAT } & \text { Poly(butylene adipate-co-terephthalate) } \\ \text { PBS } & \text { Poly(butylene succinate) } \\ \text { PCL } & \text { Poly(e-caprolactone) } \\ \text { PDLLA } & \text { Poly(d,l-lactide) } \\ \text { PEG } & \text { Poly(ethylene glycol) } \\ \text { PHA } & \text { Polyhydroxyalkanoates } \\ \text { PHEMA } & \text { Poly(2-hydroxyl ethyl methacrylate) } \\ \text { PLA } & \text { Poly(lactic acid) } \\ \text { PP } & \text { Polypropylene } \\ \text { PPC } & \text { Poly(propylene carbonate) } \\ \text { Ref. } & \text { References } \\ \text { SBM } & \text { Sleeping beauty mutase } \\ & \end{array}$

\section{References}

1. Pellis, A.; Malinconico, M.; Guarneri, A.; Gardossi, L. Renewable polymers and plastics: Performance beyond the green. New Biotechnol. 2020, 60, 146-158. [CrossRef] [PubMed]

2. Braunegg, G.; Lefebvre, G.; Genser, K.F. Polyhydroxyalkanoates, biopolyesters from renewable resources: Physiological and engineering aspects. J. Biotechnol. 1998, 65, 127-161. [CrossRef]

3. Policastro, G.; Panico, A.; Fabbricino, M. Improving biological production of poly(3-hydroxybutyrate-co-3-hydroxyvalerate) (PHBV) co-polymer: A critical review. Rev. Environ. Sci. Biotechnol. 2021, 1-35. [CrossRef] 
4. Taguchi, S.; Iwata, T.; Abe, H.; Doi, Y. 9.09-Poly(hydroxyalkanoate)s. In Polymer Science: A Comprehensive Reference; Matyjaszewski, K., Möller, M., Eds.; Elsevier: Amsterdam, The Netherlands, 2012; pp. 157-182. [CrossRef]

5. Alvarez, H.M.; Kalscheuer, R.; Steinbüchel, A. Accumulation of storage lipids in species of Rhodococcus and Nocardia and effect of inhibitors and polyethylene glycol. Lipid/Fett 1997, 99, 239-246. [CrossRef]

6. Anderson, A.J.; Williams, D.R.; Dawes, E.A.; Ewing, D.F. Biosynthesis of poly(3-hydroxybutyrate-co-3-hydroxyvalerate) in Rhodococcus ruber. Can. J. Microbiol. 1995, 41, 4-13. [CrossRef]

7. Haywood, G.W.; Anderson, A.J.; Williams, D.R.; Dawes, E.A.; Ewing, D.F. Accumulation of a poly(hydroxyalkanoate) copolymer containing primarily 3-hydroxyvalerate from simple carbohydrate substrates by Rhodococcus sp. NCIMB 40126. Int. J. Biol. Macromol. 1991, 13, 83-88. [CrossRef]

8. Valentin, H.F.; Dennis, D. Metabolic pathway for poly(3-hydroxybutyrate-co-3-hydroxyvalerate) formation in Nocardia corallina: Inactivation of mutB by chromosomal integration of a kanamycin resistance gene. Appl. Environ. Microbiol. 1996, 62, 372-379. [CrossRef] [PubMed]

9. Williams, D.R.; Anderson, A.J.; Dawes, E.A.; Ewing, D.F. Production of a co-polyester of 3-hydroxybutyric acid and 3hydroxyvaleric acid from succinic acid by Rhodococcus ruber: Biosynthetic considerations. Appl. Microbiol. Biotechnol. 1994, 40, 717-723. [CrossRef]

10. Berezina, N. Enhancing the 3-hydroxyvalerate component in bioplastic PHBV production by Cupriavidus necator. Biotechnol. J. 2012, 7, 304-309. [CrossRef]

11. Novackova, I.; Kucera, D.; Porizka, J.; Pernicova, I.; Sedlacek, P.; Koller, M.; Kovalcik, A.; Obruca, S. Adaptation of Cupriavidus necator to levulinic acid for enhanced production of $\mathrm{P}(3 \mathrm{HB}-\mathrm{co}-3 \mathrm{HV})$ copolyesters. Biochem. Eng. J. 2019, 151, 107350-107360. [CrossRef]

12. Scully, S.M.; Orlygsson, J. Biological production of alcohols. In Advanced Bioprocessing for Alternative Fuels, Biobased Chemicals, and Bioproducts; Woodhead Publishing: Cambridge, MA, USA, 2019; pp. 83-108.

13. Obruca, S.; Marova, I.; Snajdar, O.; Mravcova, L.; Svoboda, Z. Production of poly(3-hydroxybutyrate-co-3-hydroxyvalerate) by Cupriavidus necator from waste rapeseed oil using propanol as a precursor of 3-hydroxyvalerate. Biotechnol. Lett. 2010, 32, 1925-1932. [CrossRef]

14. Yamane, T.; Chen, X.; Ueda, S. Growth-associated production of poly(3-hydroxyvalerate) from $n$-pentanol by a methylotrophic bacterium, Paracoccus denitrificans. Appl. Environ. Microbiol. 1996, 62, 380-384. [CrossRef] [PubMed]

15. Strong, P.J.; Laycock, B.; Mahamud, S.N.S.; Jensen, P.D.; Lant, P.A.; Tyson, G.; Pratt, S. The opportunity for high-performance biomaterials from methane. Microorganisms 2016, 4, 11. [CrossRef] [PubMed]

16. Liu, Q.; Zhang, H.; Deng, B.; Zhao, X. Poly(3-hydroxybutyrate) and poly(3-hydroxybutyrate-co-3-hydroxyvalerate): Structure, property, and fiber. Int. J. Polym. Sci. 2014, 2014, 1-11. [CrossRef]

17. Shishatskaya, E.I.; Kamendov, I.V.; Starosvetsky, S.I.; Vinnik, Y.S.; Markelova, N.N.; Shageev, A.A.; Khorzhevsky, V.A.; Peryanova, O.V.; Shumilova, A.A. An in vivo study of osteoplastic properties of resorbable poly-3-hydroxybutyrate in models of segmental osteotomy and chronic osteomyelitis. Artif. Cells, Nanomed. Biotechnol. 2014, 42, 344-355. [CrossRef] [PubMed]

18. Naser, A.Z.; Deiab, I.; Darras, B.M. Poly(lactic acid)(PLA) and polyhydroxyalkanoates (PHAs), green alternatives to petroleumbased plastics: A review. RSC Adv. 2021, 11, 17151-17196. [CrossRef]

19. Laycock, B.; Halley, P.; Pratt, S.; Werker, A.; Lant, P. The chemomechanical properties of microbial polyhydroxyalkanoates. Prog. Polym. Sci. 2013, 38, 536-583. [CrossRef]

20. Ali, I.; Jamil, N. Polyhydroxyalkanoates: Current applications in the medical field. Front. Biol. 2016, 11, 19-27. [CrossRef]

21. Wu, L.P.; Wang, D.; Parhamifar, L.; Hall, A.; Chen, G.Q.; Moghimi, S.M. Poly(3-hydroxybutyrate-co-R-3-hydroxyhexanoate) nanoparticles with polyethylenimine coat as simple, safe, and versatile vehicles for cell targeting: Population characteristics, cell uptake, and intracellular trafficking. Adv. Healthc. Mater. 2014, 3, 817-824. [CrossRef]

22. Catoni, S.E.; Trindade, K.N.; Gomes, C.A.; Schneider, A.L.; Pezzin, A.; Soldi, V. Influence of poly(ethylene grycol)-(PEG) on the properties of influence of poly(3-hydroxybutyrate-co-3-hydroxyvalerate)-PHBV. Polimeros 2013, 23, 320-325. [CrossRef]

23. Li, Z.; Loh, X.J. Water soluble polyhydroxyalkanoates: Future materials for therapeutic applications. Chem. Soc. Rev. 2015, 44, 2865-2879. [CrossRef]

24. Shah, M.; Naseer, M.I.; Choi, M.H.; Kim, M.O.; Yoon, S.C. Amphiphilic PHA-mPEG copolymeric nanocontainers for drug delivery: Preparation, characterization and in vitro evaluation. Int. J. Pharm. 2010, 400, 165-175. [CrossRef] [PubMed]

25. Scandola, M.; Focarete, M.L.; Adamus, G.; Sikorska, W.; Baranowska, I.; Świerczek, S.; Gnatowski, M.; Kowalczuk, M.; Jedliński, Z. Polymer blends of natural poly(3-hydroxybutyrate-co-3-hydroxyvalerate) and a synthetic atactic poly(3-hydroxybutyrate). Characterization and biodegradation studies. Macromolecules 1997, 30, 2568-2574. [CrossRef]

26. Bhatia, S.K.; Wadhwa, P.; Hong, J.W.; Hong, Y.G.; Jeon, J.M.; Lee, E.S.; Yang, Y.H. Lipase mediated functionalization of poly(3hydroxybutyrate-co-3-hydroxyvalerate) with ascorbic acid into an antioxidant active biomaterial. Int. J. Biol. Macromol. 2019, 123, 117-123. [CrossRef]

27. Malmir, S.; Montero, B.; Rico, M.; Barral, L.; Bouza, R. Morphology, thermal and barrier properties of biodegradable films of poly(3-hydroxybutyrate-co-3-hydroxyvalerate) containing cellulose nanocrystals. Compos. A Appl. Sci. Manuf. 2017, 93, 41-48. [CrossRef]

28. Meereboer, K.W.; Pal, A.K.; Cisneros-López, E.O.; Misra, M.; Mohanty, A.K. The effect of natural fillers on the marine biodegradation behaviour of poly(3-hydroxybutyrate-co-3-hydroxyvalerate)(PHBV). Sci. Rep. 2021, 11, 1-11. [CrossRef] 
29. Figueroa-Lopez, K.J.; Cabedo, L.; Lagaron, J.M.; Torres-Giner, S. Development of electrospun poly(3-hydroxybutyrate-co-3hydroxyvalerate) monolayers containing eugenol and their application in multilayer antimicrobial food packaging. Front. Nutr. 2020, 7, 140-155. [CrossRef]

30. Galego, N.; Rozsa, C.; Sánchez, R.; Fung, J.; Vázquez, A.; Santo Tomás, J. Characterization and application of poly( $\beta$ hydroxyalkanoates) family as composite biomaterials. Polym. Test. 2000, 19, 485-492. [CrossRef]

31. Thiré, R.M.D.S.M.; Arruda, L.C.; Barreto, L.S. Morphology and thermal properties of poly(3-hydroxybutyrate-co-3hydroxyvalerate)/attapulgite nanocomposites. Mater. Res. 2011, 14, 340-344. [CrossRef]

32. Kwiecien, I.; Adamus, G.; Jiang, G.; Radecka, I.; Baldwin, T.C.; Khan, H.R.; Johnston, B.; Pennetta, V.; Hill, D.; Bretz, I.; et al. Biodegradable PBAT/PLA blend with bioactive MCPA-PHBV conjugate suppresses weed growth. Biomacromolecules 2018, 19, 511-520. [CrossRef]

33. Bakare, R.A.; Bhan, C.; Raghavan, D. Synthesis and characterization of collagen grafted poly(hydroxybutyrate-valerate)(PHBV) scaffold for loading of bovine serum albumin capped silver (Ag/BSA) nanoparticles in the potential use of tissue engineering application. Biomacromolecules 2014, 15, 423-435. [CrossRef] [PubMed]

34. Zhao, X.; Cornish, K.; Vodovotz, Y. Synergistic mechanisms underlie the peroxide and coagent improvement of natural-rubbertoughened poly(3-hydroxybutyrate-co-3-hydroxyvalerate) mechanical performance. Polymers 2019, 11, 565. [CrossRef] [PubMed]

35. Javadi, A.; Kramschuster, A.J.; Pilla, S.; Lee, J.; Gong, S.; Turng, L.S. Processing and characterization of microcellular PHBV/PBAT blends. Poly. Eng. Sci. 2010, 50, 1440-1448. [CrossRef]

36. Qiu, Z.; Ikehara, T.; Nishi, T. Miscibility and crystallization behaviour of biodegradable blends of two aliphatic polyesters. Poly(3-hydroxybutyrate-co-hydroxyvalerate) and poly(butylene succinate) blends. Polymer 2003, 44, 7519-7527. [CrossRef]

37. Ma, P.; Hristova-Bogaerds, D.G.; Lemstra, P.J.; Zhang, Y.; Wang, S. Toughening of PHBV/PBS and PHB/PBS blends via in situ compatibilization using dicumyl peroxide as a free-radical grafting initiator. Macromol. Mater. Eng. 2012, 297, 402-410. [CrossRef]

38. Chun, Y.S.; Kim, W.N. Thermal properties of poly(hydroxybutyrate-co-hydroxyvalerate) and poly( $\varepsilon$-caprolactone) blends. Polymer 2000, 41, 2305-2308. [CrossRef]

39. Wang, S.; Ma, P.; Wang, R.; Wang, S.; Zhang, Y.; Zhang, Y. Mechanical, thermal and degradation properties of poly(d,1lactide)/poly(hydroxybutyrate-co-hydroxyvalerate)/poly(ethylene glycol) blend. Polym. Degrad. Stab. 2008, 93, 1364-1369. [CrossRef]

40. Pillai, A.B.; Kumar, A.J.; Kumarapillai, H. Biosynthesis of poly(3-hydroxybutyrate-co-3-hydroxyvalerate) (PHBV) in Bacillus aryabhattai and cytotoxicity evaluation of PHBV/poly(ethylene glycol) blends. 3 Biotech 2020, 10, 1-10. [CrossRef]

41. Silva, A.P.B.; Montagna, L.S.; Passador, F.R.; Rezende, M.C.; Lemes, A.P. Biodegradable nanocomposites based on PLA/PHBV blend reinforced with carbon nanotubes with potential for electrical and electromagnetic applications. Express Polym. Lett. 2021, 15, 1-12. [CrossRef]

42. Zhao, H.; Cui, Z.; Wang, X.; Turng, L.S.; Peng, X. Processing and characterization of solid and microcellular poly(lactic acid)/polyhydroxybutyrate-valerate (PLA/PHBV) blends and PLA/PHBV/Clay nanocomposites. Compos. B Eng. 2013, 51, 79-91. [CrossRef]

43. Tao, J.; Song, C.; Cao, M.; Hu, D.; Liu, L.; Liu, N.; Wang, S. Thermal properties and degradability of poly(propylene carbonate)/poly( $\beta$-hydroxybutyrate-co- $\beta$-hydroxyvalerate) (PPC/PHBV) blends. Polym. Degrad. Stab. 2009, 94, 575-583. [CrossRef]

44. Syahirah, W.N.; Azami, N.A.; Huong, K.H.; Amirul, A.A. Preparation, characterization and biodegradation of blend films of poly(3-hydroxybutyrate-co-3-hydroxyvalerate) with natural biopolymers. Polym. Bull. 2020, 78, 3973-3993. [CrossRef]

45. Castro-Mayorga, J.L.; Fabra, M.J.; Pourrahimi, A.M.; Olsson, R.T.; Lagaron, J.M. The impact of zinc oxide particle morphology as an antimicrobial and when incorporated in poly(3-hydroxybutyrate-co-3-hydroxyvalerate) films for food packaging and food contact surfaces applications. Food Bioprod. Process. 2017, 101, 32-44. [CrossRef]

46. Shuai, C.; Wang, C.; Qi, F.; Peng, S.; Yang, W.; He, C.; Wang, G.; Qian, G. Enhanced crystallinity and antibacterial of PHBV scaffolds incorporated with zinc oxide. J. Nanomater. 2020, 2020,1-12. [CrossRef]

47. Alsabri, A.; Tahir, F.; Al-Ghamdi, S.G. Environmental impacts of polypropylene (PP) production and prospects of its recycling in the GCC region. Mat. Today Proc. 2021; in press. [CrossRef]

48. Sen, S.K.; Raut, S. Microbial degradation of low density polyethylene (LDPE): A review. J. Environ. Chem. Eng. 2015, 3, 462-473. [CrossRef]

49. Kader, M.A.; Senge, M.; Mojid, M.A.; Ito, K. Recent advances in mulching materials and methods for modifying soil environment. Soil Tillage Res. 2017, 168, 155-166. [CrossRef]

50. Sarkar, D.J.; Barman, M.; Bera, T.; De, M.; Chatterjee, D. Agriculture: Polymers in crop production mulch and fertilizer. In Encyclopedia of Polymer Applications; Routledge: England, UK, 2018; Volume 1, pp. 1-20.

51. Philip, S.; Keshavarz, T.; Roy, I. Polyhydroxyalkanoates: Biodegradable polymers with a range of applications. J. Chem. Technol. Biotechnol. 2007, 82, 233-247. [CrossRef]

52. Yogesh, C.; Pathak, B.; Fulekar, M.H. PHA-production application and its bioremediation in environment. Res. J. Environ. Sci. 2012, 1, 46-52. Available online: http:/ / www.isca.in/IJENS/Archive/v1/i2/9.ISCA-JEvsS-2012-009.pdf (accessed on 15 January 2022).

53. Krasnits, E.; Beliavsky, M.; Tarre, S.; Green, M. PHA based denitrification: Municipal wastewater vs. acetate. Bioresour. Technol. 2013, 132, 28-37. [CrossRef] 
54. Santorio, S.; Fra-Vázquez, A.; Del Rio, A.V.; Mosquera-Corral, A. Potential of endogenous PHA as electron donor for denitrification. Sci. Total Environ. 2019, 695, 133747-133753. [CrossRef] [PubMed]

55. Hiraishi, A.; Khan, S.T. Application of polyhydroxyalkanoates for denitrification in water and wastewater treatment. Appl. Microbiol. Biotechnol. 2003, 61, 103-109. [CrossRef] [PubMed]

56. Schulz, H. Beta oxidation of fatty acids. Biochim. Biophys. Acta Lipids Lipid Metab. 1991, 1081, 109-120. [CrossRef]

57. Azira, T.F.; Nursolehah, A.A.; Norhayati, Y.; Majid, M.I.A.; Amirul, A.A. Biosynthesis of poly(3-hydroxybutyrate-co-3hydroxyvalerate-co-4-hydroxybutyrate) terpolymer by Cupriavidus sp. USMAA2-4 through two-step cultivation process. World J. Microbiol. Biotechnol. 2011, 27, 2287-2295. [CrossRef]

58. Chen, Q.; Wang, Q.; Wei, G.; Liang, Q.; Qi, Q. Production in Escherichia coli of poly(3-hydroxybutyrate-co-3-hydroxyvalerate) with differing monomer compositions from unrelated carbon sources. Appl. Environ. Microbiol. 2011, 77, 4886-4893. [CrossRef]

59. Huijberts, G.N.; Eggink, G.; De Waard, P.; Huisman, G.W.; Witholt, B. Pseudomonas putida KT2442 cultivated on glucose accumulates poly(3-hydroxyalkanoates) consisting of saturated and unsaturated monomers. Appl. Environ. Microbiol. 1992, 58, 536-544. [CrossRef] [PubMed]

60. Majid, M.I.A.; Akmal, D.H.; Few, L.L.; Agustien, A.; Toh, M.S.; Samian, M.R.; Najimudin, N.; Azizan, M.N. Production of poly(3-hydroxybutyrate) and its copolymer poly(3-hydroxybutyrate-co-3-hydroxyvalerate) by Erwinia sp. USMI-20. Int. J. Biol. Macromol. 1999, 25, 95-104. [CrossRef]

61. Bingham, E.; Cohrssen, B.; Powell, C.H. Patty's Toxicology Volume 1-6, 6th ed.; John Wiley \& Sons: New York, NY, USA, 2012.

62. Klabunde, J.; Bischoff, C.; Papa, A.J. Propanols. In Ullmann's Encyclopedia of Industrial Chemistry; Wiley: Hoboken, NJ, USA, 2018; pp. 1-14. Available online: https:/ / onlinelibrary.wiley.com/doi/10.1002/14356007.a22_173.pub3 (accessed on 15 January 2022).

63. Mallat, T.; Baiker, A. Oxidation of alcohols with molecular oxygen on solid catalysts. Chem. Rev. 2004, 104, 3037-3058. [CrossRef]

64. Steinbüchel, A.; Lütke-Eversloh, T. Metabolic engineering and pathway construction for biotechnological production of relevant polyhydroxyalkanoates in microorganisms. Biochem. Eng. J. 2003, 16, 81-96. [CrossRef]

65. Berezina, N.; Yada, B. Improvement of the poly(3-hydroxybutyrate-co-3-hydroxyvalerate)(PHBV) production by dual feeding with levulinic acid and sodium propionate in Cupriavidus necator. New Biotechnol. 2016, 33, 231-236. [CrossRef]

66. Chung, S.H.; Park, G.G.; Kim, H.W.; Rhee, Y.H. Effect of levulinic acid on the production of poly(3-hydroxybutyrate-co-3hydroxyvalerate) by Ralstonia eutropha KHB-8862. J. Microbiol. 2001, 39, 79-82.

67. Kim, D.Y.; Park, D.S.; Kwon, S.B.; Chung, M.G.; Bae, K.S.; Park, H.Y.; Rhee, Y.H. Biosynthesis of poly(3-hydroxybutyrate-co-3hydroxyvalerate) copolyesters with a high molar fraction of 3-hydroxyvalerate by an insect-symbiotic Burkholderia sp. IS-01. J. Microbiol. 2009, 47, 651-656. [CrossRef] [PubMed]

68. Koller, M.; Hesse, P.; Fasl, H.; Stelzer, F.; Braunegg, G. Study on the effect of levulinic acid on whey-based biosynthesis of poly(3-hydroxybutyrate-co-3-hydroxyvalerate) by Hydrogenophaga pseudoflava. Appl. Food Biotechnol. 2017, 4, 65-78. [CrossRef]

69. Park, S.K.; Lee, K.T.; Kim, Y.B.; Rhee, Y.H. Biosynthesis of polyhydroxybutyrate and poly(3-hydroxybutyrate-co-3-hydroxyvalerate) by Bacillus thuringiensis R-510. J. Microbiol. 1997, 35, 127-133.

70. Gahlawat, G.; Soni, S.K. Valorization of waste glycerol for the production of poly(3-hydroxybutyrate) and poly(3-hydroxybutyrateco-3-hydroxyvalerate) copolymer by Cupriavidus necator and extraction in a sustainable manner. Bioresour. Technol. 2017, 243, 492-501. [CrossRef]

71. Grousseau, E.; Blanchet, E.; Déléris, S.; Albuquerque, M.G.; Paul, E.; Uribelarrea, J.L. Phosphorus limitation strategy to increase propionic acid flux towards 3-hydroxyvaleric acid monomers in Cupriavidus necator. Bioresour. Technol. 2014, 153, 206-215. [CrossRef]

72. Khanna, S.; Srivastava, A.K. Production of poly(3-hydroxybutyric-co-3-hydroxyvaleric acid) having a high hydroxyvalerate content with valeric acid feeding. J. Ind. Microbiol. Biotechnol. 2007, 34, 457-461. [CrossRef]

73. Kim, B.S.; Lee, S.C.; Lee, S.Y.; Chang, H.N.; Chang, Y.K.; Woo, S.I. Production of poly(3-hydroxybutyric-co-3-hydroxyvaleric acid) by fed-batch culture of Alcaligenes eutrophus with substrate control using on-line glucose analyzer. Enzym. Microb. Technol. 1994, 16, 556-561. [CrossRef]

74. Dionisi, D.; Majone, M.; Papa, V.; Beccari, M. Biodegradable polymers from organic acids by using activated sludge enriched by aerobic periodic feeding. Biotechnol. Bioeng. 2004, 85, 569-579. [CrossRef] [PubMed]

75. Das, R.; Saha, N.R.; Pal, A.; Chattopadhyay, D.; Paul, A.K. Comparative evaluation of physicochemical characteristics of biopolyesters $\mathrm{P}(3 \mathrm{HB})$ and $\mathrm{P}(3 \mathrm{HB}-\mathrm{co}-3 \mathrm{HV})$ produced by endophytic Bacillus cereus RCL 02. Front. Biol. 2018, 13, $297-308$. [CrossRef]

76. Amirul, A.A.; Syairah, S.N.; Yahya, A.R.; Azizan, M.N.M.; Majid, M.I.A. Synthesis of biodegradable polyesters by Gram-negative bacterium isolated from Malaysian environment. World J. Microbiol. Biotechnol. 2008, 24, 1327-1332. [CrossRef]

77. Kim, S.W.; Kim, P.; Kim, J.H. Production of poly(3-hydroxybutyrate-co-3-hydroxyvalerate) from Methylobacterium organophilum by potassium-limited fed-batch culture. Enzym. Microb. Technol. 1999, 24, 555-560. [CrossRef]

78. Sheu, D.S.; Chen, W.M.; Yang, J.Y.; Chang, R.C. Thermophilic bacterium Caldimonas taiwanensis produces poly(3-hydroxybutyrateco-3-hydroxyvalerate) from starch and valerate as carbon sources. Enzym. Microb. Technol. 2009, 44, 289-294. [CrossRef]

79. Myung, J.; Galega, W.M.; Van Nostrand, J.D.; Yuan, T.; Zhou, J.; Criddle, C.S. Long-term cultivation of a stable Methylocystisdominated methanotrophic enrichment enabling tailored production of poly(3-hydroxybutyrate-co-3-hydroxyvalerate). Bioresour. Technol. 2015, 198, 811-818. [CrossRef] 
80. Amini, M.; Sobhani, S.; Younesi, H.; Abyar, H.; Salamatinia, B.; Mohammadi, M. Evaluating the feasibility of poly(3hydroxybutyrate-co-3-hydroxyvalerate) co-biopolymer production from rice wastewater by Azohydromonas lata. Appl. Food Biotechnol. 2020, 7, 73-83. [CrossRef]

81. Matsumoto, K.I.; Kitagawa, K.; Jo, S.J.; Song, Y.; Taguchi, S. Production of poly(3-hydroxybutyrate-co-3-hydroxyvalerate) in recombinant Corynebacterium glutamicum using propionate as a precursor. J. Biotechnol. 2011, 152, 144-146. [CrossRef] [PubMed]

82. Doi, Y.; Kunioka, M.; Nakamura, Y.; Soga, K. Biosynthesis of copolyesters in Alcaligenes eutrophus H16 from carbon-13 labeled acetate and propionate. Macromolecules 1987, 20, 2988-2991. [CrossRef]

83. Bhubalan, K.; Lee, W.H.; Loo, C.Y.; Yamamoto, T.; Tsuge, T.; Doi, Y.; Sudesh, K. Controlled biosynthesis and characterization of poly(3-hydroxybutyrate-co-3-hydroxyvalerate-co-3-hydroxyhexanoate) from mixtures of palm kernel oil and 3HV-precursors. Polym. Degrad. Stab. 2008, 93, 17-23. [CrossRef]

84. Catalán, A.I.; Malan, A.K.; Ferreira, F.; Gill, P.R.; Batista, S. Propionic acid metabolism and poly-3-hydroxybutyrate-co-3hydroxyvalerate production by a prpC mutant of Herbaspirillum seropedicae Z69. J. Biotechnol. 2018, 286, 36-44. [CrossRef]

85. Lee, W.H.; Loo, C.Y.; Nomura, C.T.; Sudesh, K. Biosynthesis of polyhydroxyalkanoate copolymers from mixtures of plant oils and 3-hydroxyvalerate precursors. Bioresour. Technol. 2008, 99, 6844-6851. [CrossRef]

86. Cal, A.J.; Sikkema, W.D.; Ponce, M.I.; Franqui-Villanueva, D.; Riiff, T.J.; Orts, W.J.; Pieja, A.J.; Lee, C.C. Methanotrophic production of polyhydroxybutyrate-co-hydroxyvalerate with high hydroxyvalerate content. Int. J. Biol. Macromol. 2016, 87, 302-307. [CrossRef] [PubMed]

87. Wong, H.S.J.; Huong, K.H.; Hani, S.N.A.; Amirul, A.A.A. Genetic incorporation of oil-utilizing ability in Cupriavidus malaysiensis USMAA2-4 for sustainable polyhydroxyalkanoates production from palm olein and 1-pentanol. J. Biotechnol. 2021, 337, 71-79. [CrossRef] [PubMed]

88. Huong, K.H.; Shantini, K.; Sharmini, R.; Amirul, A.A. Exploring the potential of 1-pentanol and oleic acid for optimizing the production of poly(3-hydroxybutyrate-co-3-hydroxyvalerate) copolymer by Cupriavidus sp. USMAA1020. Arab. J. Sci. Eng. 2017, 42, 2313-2320. [CrossRef]

89. Kiun, J.T.; Amelia, T.S.M.; Huong, K.H.; Amirul, A.A.; Bhubalan, K. Optimizing the biosynthesis of renewable polyhydroxyalkanoate copolymer containing 3-hydroxyvalerate by Massilia haematophila using statistical modeling. BioTechnologia 2019, 100, 359-371. [CrossRef]

90. Ezhov, V.A.; Doronina, N.V.; Trotsenko, Y.A. Biosynthesis of polyhydroxybutyrate/valerate with different molecular weights during the growth of Methylobacterium extorquens G-10 on a methanol-pentanol mixture. Appl. Biochem. Microbiol. 2013, 49, 150-153. [CrossRef]

91. Galuzina, T.V.; Gerasin, V.A.; Doronina, N.V.; Ezhov, V.A.; Trotsenko, Y.A.; Kiprianov, S.V.; Ivanov, A.O.; Filatova, M.P.; Shklyaruk, B.F. Structures and properties of polyhydroxyalkanoates synthesized by Methyloligella halotolerans $\mathrm{C} 2$ and Methylobacterium extorquens G10 from a methanol-pentanol mixture. Polym. Sci. Ser. A 2015, 57, 729-737. [CrossRef]

92. Raberg, M.; Voigt, B.; Hecker, M.; Steinbüchel, A. A closer look on the polyhydroxybutyrate-(PHB)-negative phenotype of Ralstonia eutropha PHB-4. PLoS ONE 2014, 9, e95907. [CrossRef]

93. Choi, J.; Lee, S.Y. Factors affecting the economics of polyhydroxyalkanoate production by bacterial fermentation. Appl. Microbiol. Biotechnol. 1999, 51, 13-21. [CrossRef]

94. Saratale, R.G.; Cho, S.K.; Saratale, G.D.; Kadam, A.A.; Ghodake, G.S.; Kumar, M.; Bharagava, R.N.; Kumar, G.; Kim, D.S.; Mulla, S.I.; et al. A comprehensive overview and recent advances on polyhydroxyalkanoates (PHA) production using various organic waste streams. Bioresour. Technol. 2021, 325, 124685-124699. [CrossRef] [PubMed]

95. Bhattacharyya, A.; Jana, K.; Haldar, S.; Bhowmic, A.; Mukhopadhyay, U.K.; De, S.; Mukherjee, J. Integration of poly-3(hydroxybutyrate-co-hydroxyvalerate) production by Haloferax mediterranei through utilization of stillage from rice-based ethanol manufacture in India and its techno-economic analysis. World J. Microbiol. Biotechnol. 2015, 31, 717-727. [CrossRef] [PubMed]

96. Trzeciak, A.M. 6.02-Hydroformylation. In Comprehensive Inorganic Chemistry II, 2nd ed.; Reedijk, J., Poeppelmeier, K., Eds.; Elsevier: Amsterdam, The Netherlands, 2013; pp. 25-46. [CrossRef]

97. Torres, G.M.; Frauenlob, R.; Franke, R.; Börner, A. Production of alcohols via hydroformylation. Catal. Sci. Technol. 2015, 5, 34-54. [CrossRef]

98. Molnár, Á.; Papp, A. Catalyst recycling-a survey of recent progress and current status. Coord. Chem. Rev. 2017, 349, 1-65. [CrossRef]

99. Lappe, P.; Hofmann, T. Pentanols. In Ullmann's Encyclopedia of Industrial Chemistry; Wiley: Hoboken, NJ, USA, 2012; pp. 245-258. Available online: https:/ / onlinelibrary.wiley.com/doi/10.1002/14356007.a19_049.pub2 (accessed on 15 January 2022).

100. Cropley, J.B.; Burgess, L.M.; Loke, R.A. Butyraldehyde hydrogenation-a case-study in process design. Chemtech 1984, 14, 374-380.

101. Faith, W.L.; Keyes, D.B.; Clark, R.L. Industrial Chemicals, 3rd ed.; Wiley \& Sons: New York, NY, USA, 1965.

102. Fuchs, D.; Rousseau, G.; Diab, L.; Gellrich, U.; Breit, B. Tandem rhodium-catalyzed hydroformylation-hydrogenation of alkenes by employing a cooperative ligand system. Angew. Chem. Int. Ed. 2012, 51, 2178-2182. [CrossRef] [PubMed]

103. Allen, S.H.G.; Kellermeyer, R.W.; Stjernholm, R.L.; Wood, H.G. Purification and properties of enzymes involved in the propionic acid fermentation. J. Bacteriol. 1964, 87, 171-187. [CrossRef]

104. Wood, H.G. Metabolic cycles in the fermentation by propionic acid bacteria. In Current Topics in Cellular Regulation; Academic Press: Cambridge, MA, USA, 1981; Volume 18, pp. 255-287. [CrossRef] 
105. Himmi, E.H.; Bories, A.; Boussaid, A.; Hassani, L. Propionic acid fermentation of glycerol and glucose by Propionibacterium acidipropionici and Propionibacterium freudenreichii ssp. shermanii. Appl. Microbiol. Biotechnol. 2000, 53, 435-440. [CrossRef] [PubMed]

106. Seeliger, S.; Janssen, P.H.; Schink, B. Energetics and kinetics of lactate fermentation to acetate and propionate via methylmalonylCoA or acrylyl-CoA. FEMS Microbiol. Lett. 2002, 211, 65-70. [CrossRef] [PubMed]

107. Walther, T.; François, J.M. Microbial production of propanol. Biotechnol. Adv. 2016, 34, 984-996. [CrossRef]

108. Cardon, B.P.; Barker, H.A. Two new amino-acid-fermenting bacteria, Clostridium propionicum and Diplococcus glycinophilus. J. Bacterial. 1946, 52, 629-634. [CrossRef]

109. Tholozan, J.L.; Touzel, J.P.; Samain, E.; Grivet, J.P.; Prensier, G.; Albagnac, G. Clostridium neopropionicum sp. nov., a strict anaerobic bacterium fermenting ethanol to propionate through acrylate pathway. Arch. Microbiol. 1992, 157, 249-257. [CrossRef]

110. Liu, K.; Atiyeh, H.K.; Stevenson, B.S.; Tanner, R.S.; Wilkins, M.R.; Huhnke, R.L. Continuous syngas fermentation for the production of ethanol, n-propanol and n-butanol. Bioresour. Technol. 2014, 151, 69-77. [CrossRef] [PubMed]

111. Millat, T.; Janssen, H.; Thorn, G.J.; King, J.R.; Bahl, H.; Fischer, R.J.; Wolkenhauer, O. A shift in the dominant phenotype governs the $\mathrm{pH}$-induced metabolic switch of Clostridium acetobutylicum in phosphate-limited continuous cultures. Appl. Microbiol. Biotechnol. 2013, 97, 6451-6466. [CrossRef] [PubMed]

112. Isom, C.E.; Nanny, M.A.; Tanner, R.S. Improved conversion efficiencies for $n$-fatty acid reduction to primary alcohols by the solventogenic acetogen "Clostridium ragsdalei". J. Ind. Microbiol. Biotechnol. 2015, 42, 29-38. [CrossRef] [PubMed]

113. Atsumi, S.; Liao, J.C. Directed evolution of Methanococcus jannaschii citramalate synthase for biosynthesis of 1-propanol and 1-butanol by Escherichia coli. Appl. Environ. Microbiol. 2008, 74, 7802-7808. [CrossRef] [PubMed]

114. Shen, C.R.; Liao, J.C. Metabolic engineering of Escherichia coli for 1-butanol and 1-propanol production via the keto-acid pathways. Metab. Eng. 2008, 10, 312-320. [CrossRef]

115. Shen, C.R.; Liao, J.C. Synergy as design principle for metabolic engineering of 1-propanol production in Escherichia coli. Metab. Eng. 2013, 17, 12-22. [CrossRef]

116. Chen, G.S.; Siao, S.W.; Shen, C.R. Saturated mutagenesis of ketoisovalerate decarboxylase V461 enabled specific synthesis of 1-pentanol via the ketoacid elongation cycle. Sci. Rep. 2017, 7, 1-12. [CrossRef]

117. Haller, T.; Buckel, T.; Rétey, J.; Gerlt, J.A. Discovering new enzymes and metabolic pathways: Conversion of succinate to propionate by Escherichia coli. Biochemistry 2000, 39, 4622-4629. [CrossRef]

118. Srirangan, K.; Liu, X.; Westbrook, A.; Akawi, L.; Pyne, M.E.; Moo-Young, M.; Chou, C.P. Biochemical, genetic, and metabolic engineering strategies to enhance coproduction of 1-propanol and ethanol in engineered Escherichia coli. Appl. Microbiol. Biotechnol. 2015, 98, 9499-9515. [CrossRef]

119. Park, H.; Jeon, B.S.; Sang, B.I. efficient, simple production of corresponding alcohols from supplemented $\mathrm{C}_{2}-\mathrm{C}_{8}$ carboxylic acids in Escherichia coli using acyl-CoA transferase from Megasphaera hexanoica. Biotechnol. Bioprocess. Eng. 2020, 25, 599-606. [CrossRef]

120. Steinbüchel, A.; Schlegel, H.G. Physiology and molecular genetics of poly( $\beta$-hydroxyalkanoic acid) synthesis in Alcaligenes eutrophus. Mol. Microbiol. 1991, 5, 535-542. [CrossRef] [PubMed]

121. Kelly, D.P.; Rainey, F.A.; Wood, A.P. The genus Paracoccus. In The Prokaryotes; Dworkin, M., Falkow, S., Rosenberg, E., Schleifer, K.H., Stackebrandt, E., Eds.; Springer: New York, NY, USA, 2006; pp. 232-249. [CrossRef]

122. Akmal, D.; Asiska, P.D.; Wangi, Q.A.; Rivai, H.; Agustien, A. Biosynthesis of copolymer poly(3-hydroxybutyrate-co-3hydroxyvalerate) from palm oil and $n$-pentanol in a 10 L bioreactor. Rasayan J. Chem. 2015, 8, 389-395.

123. Amirul, A.A.; Yahya, A.R.; Sudesh, K.; Azizan, M.N.M.; Majid, M.I.A. Isolation of poly(3-hydroxybutyrate-co-4-hydroxybutyrate) producer from Malaysian environment using $\gamma$-butyrolactone as carbon source. World J. Microbiol. Biotechnol. 2009, 25, 1199-1206. [CrossRef]

124. Ramachandran, H.; Shafie, N.A.H.; Sudesh, K.; Azizan, M.N.; Majid, M.I.A.; Amirul, A.A.A. Cupriavidus malaysiensis sp. nov.; a novel poly(3-hydroxybutyrate-co-4-hydroxybutyrate) accumulating bacterium isolated from the Malaysian environment. Antonie Leeuwenhoek 2018, 111, 361-372. [CrossRef] [PubMed]

125. Shantini, K.; Bhubalan, K.; Yahya, A.R.M.; Amirul, A.A. Productivity increment of biodegradable and biorenewable copolymer containing 3-hydroxyvalerate monomer initiated by alcohols as precursor substrates. J. Chem. Technol. Biotechnol. 2013, 88, 1364-1370. [CrossRef]

126. Sashi, P.; Yasin, U.M.; Bhuyan, A.K. Unfolding action of alcohols on a highly negatively charged state of cytochrome c. Biochemistry 2012, 51, 3273-3283. [CrossRef]

127. Ingram, L.O. Adaptation of membrane lipids to alcohols. J. Bacterial. 1976, 125, 670-678. [CrossRef]

128. Jovanovic, G.; Lloyd, L.J.; Stumpf, M.P.; Mayhew, A.J.; Buck, M. Induction and function of the phage shock protein extracytoplasmic stress response in Escherichia coli. J. Biol. Chem. 2006, 281, 21147-21161. [CrossRef]

129. Yano, T.; Miyahara, Y.; Morii, N.; Okano, T.; Kubota, H. Pentanol and benzyl alcohol attack bacterial surface structures differently. Appl. Environ. Microbiol. 2016, 82, 402-408. [CrossRef]

130. Osman, Y.A.; Ingram, L.O. Mechanism of ethanol inhibition of fermentation in Zymomonas mobilis CP4. J. Bacterial. 1985, 164, 173-180. [CrossRef]

131. Ingram, L.O.N.; Buttke, T.M. Effects of alcohols on micro-organisms. Adv. Microb. Physiol. 1985, 25, 253-300. [CrossRef] 
132. Heipieper, H.J.; Meinhardt, F.; Segura, A. The cis-trans isomerase of unsaturated fatty acids in Pseudomonas and Vibrio: Biochemistry, molecular biology and physiological function of a unique stress adaptive mechanism. FEMS Microbiol. Lett. 2003, $229,1-7$. [CrossRef]

133. Beaven, M.J.; Charpentier, C.; Rose, A.H. Production and tolerance of ethanol in relation to phospholipid fatty-acyl composition in Saccharomyces cerevisiae NCYC 431. Microbiology 1982, 128, 1447-1455. [CrossRef]

134. Kitahara, K.; Takichi, K.; Osamu, G. Taxonomic studies on the hiochi-bacteria, specific saprophytes of sake II. Identification and classification of hiochi-bacteria. J. Gen. Appl. Microbiol. 1957, 3, 111-120. [CrossRef]

135. Bernal, P.; Muñoz-Rojas, J.; Hurtado, A.; Ramos, J.L.; Segura, A. A Pseudomonas putida cardiolipin synthesis mutant exhibits increased sensitivity to drugs related to transport functionality. Environ. Microbiol. 2007, 9, 1135-1145. [CrossRef] [PubMed]

136. Marr, A.G.; Ingraham, J.L. Effect of temperature on the composition of fatty acids in Escherichia coli. J. Bacteriol. 1962, 84, 1260-1267. [CrossRef] [PubMed]

137. Brynildsen, M.P.; Liao, J.C. An integrated network approach identifies the isobutanol response network of Escherichia coli. Mol. Syst. Biol. 2009, 5, 277-279. [CrossRef] [PubMed]

138. Davidson, A.L.; Dassa, E.; Orelle, C.; Chen, J. Structure, function, and evolution of bacterial ATP-binding cassette systems. Microbiol. Mol. Biol. Rev. 2008, 72, 317-364. [CrossRef]

139. Rutherford, B.J.; Dahl, R.H.; Price, R.E.; Szmidt, H.L.; Benke, P.I.; Mukhopadhyay, A.; Keasling, J.D. Functional genomic study of exogenous n-butanol stress in Escherichia coli. Appl. Environ. Microbiol. 2010, 76, 1935-1945. [CrossRef]

140. Hews, C.L.; Cho, T.; Rowley, G.; Raivio, T.L. Maintaining integrity under stress: Envelope stress response regulation of pathogenesis in Gram-negative bacteria. Front. Cell. Infect. Microbiol. 2019, 9, 313-337. [CrossRef]

141. Isaac, D.D.; Pinkner, J.S.; Hultgren, S.J.; Silhavy, T.J. The extracytoplasmic adaptor protein CpxP is degraded with substrate by DegP. Proc. Natl. Acad. Sci. USA 2005, 102, 17775-17779. [CrossRef] [PubMed]

142. Mitra, R.; Gadkari, V.V.; Meinen, B.A.; van Mierlo, C.P.; Ruotolo, B.T.; Bardwell, J.C. Mechanism of the small ATP-independent chaperone Spy is substrate specific. Nat. Commun. 2021, 12, 1-13. [CrossRef] [PubMed]

143. Tomas, C.A.; Welker, N.E.; Papoutsakis, E.T. Overexpression of groESL in Clostridium acetobutylicum results in increased solvent production and tolerance, prolonged metabolism, and changes in the cell's transcriptional program. Appl. Environ. Microbiol. 2003, 69, 4951-4965. [CrossRef] [PubMed]

144. Postma, P.W.; Lengeler, J.W.; Jacobson, G.R. Phosphoenolpyruvate: Carbohydrate phosphotransferase systems of bacteria. Microbiol Rev. 1993, 57, 543-594. [CrossRef] [PubMed]

145. Igarashi, K.; Fujita, N.; Ishihama, A. Sequence analysis of two temperature-sensitive mutations in the alpha subunit gene (rpoA) of Escherichia coli RNA polymerase. Nucleic Acids Res. 1990, 18, 5945-5948. [CrossRef] [PubMed]

146. Jafri, S.; Urbanowski, M.L.; Stauffer, G.V. A mutation in the $r p o A$ gene encoding the alpha subunit of RNA polymerase that affects metE-metR transcription in Escherichia coli. J. Bacteriol. 1995, 177, 524-529. [CrossRef]

147. Klein-Marcuschamer, D.; Santos, C.N.S.; Yu, H.; Stephanopoulos, G. Mutagenesis of the bacterial RNA polymerase alpha subunit for improvement of complex phenotypes. Appl. Environ. Microbiol. 2009, 75, 2705-2711. [CrossRef] [PubMed]

148. Peck, M.C.; Gaal, T.; Fisher, R.F.; Gourse, R.L.; Long, S.R. The RNA polymerase $\alpha$ subunit from Sinorhizobium meliloti can assemble with RNA polymerase subunits from Escherichia coli and function in basal and activated transcription both in vivo and in vitro. J. Bacteriol. 2002, 184, 3808-3814. [CrossRef] [PubMed]

149. Thomas, M.S.; Glass, R.E. Escherichia coli rpoA mutation which impairs transcription of positively regulated systems. Mol. Microbiol. 1991, 5, 2719-2725. [CrossRef] [PubMed]

150. Kidwell, J.; Valentin, H.E.; Dennis, D. Regulated expression of the Alcaligenes eutrophus pha biosynthesis genes in Escherichia coli. Appl. Environ. Microbiol. 1995, 61, 1391-1398. [CrossRef]

151. Nomura, C.T.; Taguchi, K.; Taguchi, S.; Doi, Y. Coexpression of genetically engineered 3-ketoacyl-ACP synthase III (fabH) and polyhydroxyalkanoate synthase ( $\mathrm{haC}$ ) genes leads to short-chain-length-medium-chain-length polyhydroxyalkanoate copolymer production from glucose in Escherichia coli JM109. Appl. Environ. Microbiol. 2004, 70, 999-1007. [CrossRef] [PubMed]

152. Taguchi, K.; Aoyagi, Y.; Matsusaki, H.; Fukui, T.; Doi, Y. Over-expression of 3-ketoacyl-ACP synthase III or malonyl-CoA-ACP transacylase gene induces monomer supply for polyhydroxybutyrate production in Escherichia coli HB101. Biotechnol. Lett. 1999, 21, 579-584. [CrossRef] 